\title{
1 Variation in a pleiotropic hub gene drives morphological evolution: 2 Insights from interspecific differences in head shape and eye size in 3 Drosophila
}

Buchberger, Elisa $^{1}$; Bilen, An11 ${ }^{1}$; Ayaz, Sanem ${ }^{1}$; Salamanca, David ${ }^{1,2}$; Matas de las Heras, Cristina ${ }^{1}$; Niksic, Armin ${ }^{1}$; Almudi, Isabel ${ }^{3}$; Torres-Oliva, Montserrat ${ }^{1,4}$; Casares, Fernando ${ }^{3}$; Posnien, Nico ${ }^{1, *}$

${ }^{1}$ University Göttingen, Department of Developmental Biology, Justus-von-Liebig-Weg 11, 37077 Göttingen, Germany

2 current address: Department of Integrative Zoology, University of Vienna, Althanstraße 14, 1090 Vienna, Austria

${ }^{3}$ CABD (CSIC/UPO/JA), DMC2 Unit, Pablo de Olavide University campus, Ctra. Utrera km1, 41013 Seville, Spain

${ }^{4}$ current address: Institute of Clinical Molecular Biology, Christian-Albrechts-University of Kiel, University Hospital Schleswig-Holstein, Kiel, Germany

*To whom correspondence may be addressed. Email: nposnie@gwdg.de

\section{Keywords}

Evolutionary Hotspots, Repeated Evolution, Gene Regulatory Networks, Evolution of Development, Drosophila, eye-antennal disc

\section{Author contribution}

EB: Conceptualization, Data curation, Formal analysis, Investigation, Methodology, Visualization, Writing - original draft, Writing- review and editing. AB: Investigation. SA: Investigation. DS: Investigation. CMH: Investigation. AN: Investigation. IA: Resources, Writing - review and editing, MTO: Investigation, Resources, Writing - review and editing, FC: Investigation, Writing- review and editing. NP: Conceptualization, Funding acquisition, Project administration, Resources, Supervision, Visualization, Writing - original draft, Writing - review and editing. 


\section{Abstract}

Revealing the mechanisms underlying the breath-taking morphological diversity observed in nature is a major challenge in Biology. It has been established that recurrent mutations in hotspot genes cause the repeated evolution of rather simple morphological traits, such as body pigmentation or the gain and loss of structures. To date, however, it remains elusive whether hotspot genes contribute to natural variation in complex morphological traits, such as the size and shape of organs. Since natural variation in head morphology is pervasive in Drosophila, we studied the molecular and developmental basis of differences in compound eye size and head shape in two closely related Drosophila species. We show that differences in both traits are established late during head development and we applied comparative transcriptomics and chromatin accessibility data to identify the GATA transcription factor Pannier (Pnr) as central factor regulating these differences. Although the genetic manipulation of Pnr affected multiple aspects of dorsal head development, the effect of natural variation is restricted to a subset of the phenotypic space. We present data suggesting that this developmental constraint is caused by the co-evolution of expression of $p n r$ and its co-factor $u$ shaped (ush). We propose that natural variation in highly connected developmental regulators with pleiotropic functions is a major driver for morphological evolution and we discuss implications on gene regulatory network evolution. In comparison to previous findings, our data strongly suggests that evolutionary hotspots do not contribute to the repeated evolution of eye size and head shape in Drosophila. 


\section{Introduction}

(1)

The morphological diversity present in nature is a key prerequisite for organisms to adapt to an ever-changing environment. Since the genome of an organism contains instructive information about its morphology, one of the major goals in biological research is to establish genotype-phenotype correlations for a given morphological trait [1,2]. The genetic architecture of relatively simple traits has been successfully determined at high resolution. For instance, natural variation in body pigmentation in the vinegar fly Drosophila melanogaster and the beach mouse Peromyscus polionotus has been mapped to individual nucleotides affecting gene expression [3,4] or protein function [5], respectively. Also, the genetic basis of gain or loss of structures like trichomes in Drosophila [6], armor plates in three spine stickleback fish [7] or the repeated loss of eyes in cave fish [8,9] has been successfully revealed. Intriguingly, the analysis of similar morphological differences in various lineages showed that the same genes were repeatedly affected. For instance, differences in abdominal pigmentation and the occurrence of wing spots across the phylogeny of Drosophilidae have been mapped to the bric$\grave{a}$-brac (bab) [10] and yellow (y) [11] loci, respectively. Also the evolution of larval trichome patterns in various Drosophila species is caused by variation in the regulation of the shavenbaby $(s v b)$ gene [12] and recurrent natural variation in the Pitxl locus has been causally linked to pelvic spine reduction in different stickleback lineages [13,14]. While recurrent natural variation in such hotspot genes seems to be common for the evolution of rather simple morphological traits, it remains elusive, whether evolutionary hotspots play a major role in the evolution of complex traits, such as the size and shape of organs.

The identification of the molecular changes underlying variation in complex morphological traits is difficult because many genomic loci with small effect sizes that are spread throughout the genome contribute to trait differences [15-17]. For instance, natural variation in mandible and craniofacial shape in mouse is influenced by various loci located on most of the chromosomes [18-21] and studies in Drosophila revealed loci on several chromosomes associated with differences in eye size and head shape [22-24]. The omnigenic model suggests that most genomic loci can influence the phenotypic outcome even though they are not functionally linked to the trait ("peripheral genes") because they are connected to loci with a direct effect ("core genes") in highly wired gene regulatory networks (GRNs) [15]. As many other biological networks [25-28], GRNs are thought to be scale-free, meaning that most nodes within the network have only very few connections and these low-degree nodes are 
connected by a few highly connected nodes, so called hubs [29,30]. Genes that act as hubs relay complex incoming information to a high number of target genes and they often code for evolutionary conserved proteins with crucial functions within the network [31,32]. Therefore, it is conceivable that "core genes" proposed by the omnigenic model have features of hub genes within GRNs. It has been suggested that the recurrent evolution in hotspot genes is facilitated by their hub position within GRNs [12,33,34], as well as the observation that only few such hubs are necessary to orchestrate the formation of rather simple traits [35]. In contrast, the development of complex organs relies on the interplay of many hub genes [36,37], suggesting that evolutionary hotspots are less likely to be relevant for complex trait evolution.

An excellent model to test, whether natural variation in complex traits is caused by recurrent changes in hotspot genes is the Drosophila head that harbours major sensory organs, such as the compound eyes [38]. Natural intra- and interspecific variation in eye size is pervasive in various Drosophila lineages and increased eye size is often associated with a reduction of the interstitial cuticle between the eyes [23,39-42]. While the genetic architecture of this trade-off is starting to be revealed in various species [22-24,43], the most comprehensive genetic and developmental analysis established that intra- and interspecific differences in the trade-off are caused by variation in the early subdivision of the eye-antennal imaginal disc [44], the larval tissue from which most of the adult head develops [45]. Genetic analyses between different $D$. melanogaster strains revealed a single nucleotide polymorphism in the regulatory region of the eyeless/pax6 (ey) gene affecting its temporal expression and thus eye-antennal disc subdivision [44]. Ey regulates various target genes [46] and signalling pathways [47] to initiate retinal development, suggesting that it acts as a hub gene during eye development. Additionally, the recurrent variation in ey expression suggests that ey might also be a hotspot gene regulating the trade-off between eye and head cuticle development [44]. We sought to test, whether variation in the early subdivision of the eye-antennal disc is a common mechanism underlying the development of a head trade-off in Drosophila. As model we studied head development in the two closely related Drosophila species D. melanogaster and D. mauritiana, which vary in adult eye size and head shape $[23,42,48]$. Specifically, D. mauritiana develops larger compound eyes with more ommatidia and the bigger eyes of D. mauritiana are accompanied by a narrower interstitial head cuticle [42].

In contrast to previous reports, we could show that differences in compartment sizes occur only late during eye-antennal disc development, suggesting that the early subdivision of the disc is not affected. To reveal candidate genes responsible for eye size differences, we 
1 developed a new method to identify evolutionary relevant hub genes with crucial positions

2 within the developmental GRN. Applying comparative transcriptomics and functional genomics, we revealed the GATA transcription factor Pannier (Pnr) and its transcriptional cofactor U-shaped (Ush) as candidate hub genes. We functionally confirmed that the overexpression of pnr in D. melanogaster indeed phenocopies aspects of the D. mauritiana

\section{Results}

Differences in dorsal head shape between D. melanogaster and D. mauritiana are defined during late eye-antennal disc development

Drosophila eye size and head shape vary extensively between $D$. melanogaster and $D$. mauritiana with the latter having bigger eyes due to more ommatidia at the expense of interstitial head cuticle $[22,42,48]$. Since eye size differences are most pronounced in the dorsal part [42], we first quantified differences in the dorsal head morphology to test whether dorsal head shape varies as well. We placed 57 landmarks on pictures of dorsal heads and applied a geometric morphometrics analysis (Fig 1A). A discriminate function analysis clearly distinguished the head shapes of D. melanogaster and D. mauritiana (Fig 1B) (p-value= 0.0001). In accordance with previous data [42], we found main differences in dorsal eye size with the eye area protruding more towards the back of the head in D. mauritiana (Fig 1B). The expansion of the eye area in D. mauritiana was accompanied by a narrower dorsal head region, which affected both the orbital cuticle and the dorsal frons region. The ocellar complex was slightly shifted ventrally in D. mauritiana (Fig 1B). Therefore, D. melanogaster and D. mauritiana do not only differ in the size of dorsal eye, but they also exhibit variation in the relative contribution of different head regions to the dorsal head.

To understand the developmental basis of the size and shape differences in dorsal head structures (Fig 1B), we compared eye-antennal disc development between species at the onset (72 $\mathrm{h}$ after egg laying; AEL), progression (96 h AEL) and termination of differentiation (120 h AEL) (Fig 1C). The size of the antennal and retinal region was not significantly different at 72 
bioRxiv preprint doi: https://doi.org/10.1101/2020.01.24.918011; this version posted June 14, 2020. The copyright holder for this preprint (which was not certified by peer review) is the author/funder, who has granted bioRxiv a license to display the preprint in perpetuity. It is made available under aCC-BY-NC-ND 4.0 International license.

$1 \mathrm{~h}$ AEL, respectively (Fig 1D and 1E). First differences for both disc compartments were 2 observed at $96 \mathrm{~h} \mathrm{AEL}$ and differences were clearly visible at $120 \mathrm{~h}$ AEL. At these stages, $D$. 3 mauritiana had a bigger retinal region and a smaller antennal region compared to $D$. melanogaster (Fig 1D and 1E). The region giving rise to the interstitial cuticle showed interspecific size differences from $96 \mathrm{~h} \mathrm{AEL} \mathrm{on} \mathrm{(Fig} \mathrm{1F)} \mathrm{and} \mathrm{the} \mathrm{differences} \mathrm{in} \mathrm{the} \mathrm{size} \mathrm{of} \mathrm{the}$ retinal region was accompanied by significant differences in the number of ommatidia rows at $96 \mathrm{~h}$ and $120 \mathrm{~h}$ AEL (Fig 1G). Our data shows that the trade-off between head regions is defined late during eye-antennal disc development in D. melanogaster and D. mauritiana.
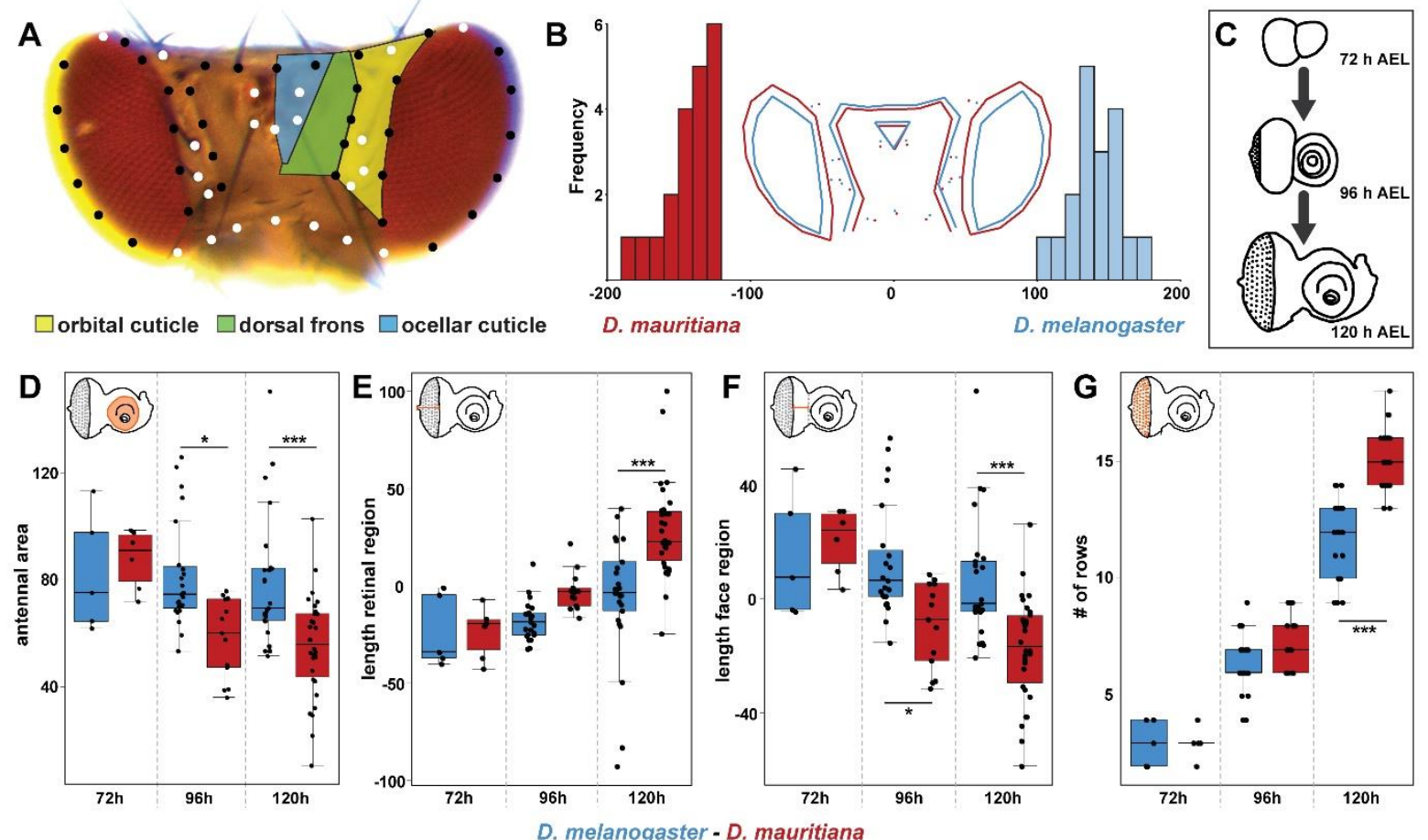

Fig 1. Quantitative differences in dorsal head shape are defined late during eye-antennal disc development. (A) Dorsal view of a Drosophila melanogaster head. The head cuticle consists of three morphologically distinguishable regions, namely the orbital cuticle (yellow) next to the compound eye, the dorsal frons (green) and the ocellary cuticle (blue). The 57 landmarks that were used to analyze head shape are shown as fixed (white) and sliding landmarks (black). (B) Discriminant function analysis distinguishes mean dorsal head shapes of D. melanogaster (blue) and D. mauritiana (red). Difference between means: Procrustes distance: 0.094, p-value $=0.0001$ (C) The development was characterized and transcriptomic datasets were generated for developing eye-antennal discs for both species at three developmental stages: 72h after egg laying (AEL; late L2), 96h AEL (mid L3) and 120h AEL (late L3). (D) Area of the antennal region of the eye-antennal disc. Significant differences were observed at $96 \mathrm{~h}$ AEL and $120 \mathrm{~h}$ AEL (F5,96 = 7.86, p = 3.08e-6). (E) Distance from the optic stalk to the morphogenetic furrow was measured along the equator region. Significant differences were observed at $120 \mathrm{~h}$ AEL (F5,96 $=15.61, \mathrm{p}=3.2 \mathrm{e}-$ 11). (F) Distance from the morphogenetic furrow to the antennal anlagen was measured. From $96 \mathrm{~h}$ AEL on, we observed significant differences $(F 5,96=10.23, p=7 e-8)$. (G) Number of ommatidial precursor rows was counted along the equator region of the eye-antennal disc. Significant differences were observed at $120 \mathrm{~h}$ after egg laying (AEL) (F5,96 = 210.8, p < 2e16). One-way ANOVA followed by Tukey multiple comparisons: $* * *<0.001 ; *<0.05$ 
Morphological differences between D. melanogaster and D. mauritiana are associated with variation in the developmental transcriptomic landscape

Next we tested whether differences in adult head morphology and eye-antennal disc development were associated with variation in the developmental transcriptomic landscape. We obtained comparative transcriptomes for eye-antennal disc development covering $72 \mathrm{~h}, 96 \mathrm{~h}$ and $120 \mathrm{~h}$ AEL (Fig 1C and S1 Fig) [49]. In line with the overall functional conservation of head morphology between species, we first confirmed that genes with stable interspecific expression represent central processes involved in eye-antennal disc development (S2 Fig). An analysis of those genes that were differentially expressed between species revealed that $72 \%$ of variation in the dataset was due to differences between $72 \mathrm{~h}$ and the other two stages (S3 Fig), an observation that was confirmed by a pairwise differential expression analysis. With 6,683 genes, we found the highest number of differentially expressed genes (DEGs) at $72 \mathrm{~h}$ AEL, while only 3,260 and 2,380 genes were differentially expressed at 96h AEL and $120 \mathrm{~h} \mathrm{AEL,}$ respectively. This excess of DEGs at $72 \mathrm{~h}$ AEL is most likely caused by the fact that is impossible to distinguish the sex of larvae at this early stage. Therefore, a mix of males and females was sequenced at $72 \mathrm{~h} \mathrm{AEL}$, while only females were analysed at the two later timepoints [49]. Differential expression was not biased towards one species since we observed an equal number of DEGs with higher expression in D. melanogaster and D. mauritiana, respectively (S1 Table).

To identify putative developmental processes affected by the interspecific expression differences, we clustered DEGs based on temporal expression profiles and then we searched for enriched GO categories associated with each gene cluster (Fig 2). Genes involved in generic metabolic and energy related processes were enriched in various clusters including those with dynamic (Fig 2, e.g. cluster 1 and 2) as well as stable temporal expression (Fig 2, e.g. cluster 11). Genes that tended to be highly expressed at later stages were enriched for factors regulating neuronal differentiation processes (Fig 2, e.g. cluster 4) and in clusters with stably expressed genes, we observed an enrichment for cell cycle control and tissue growth (Fig 2, e.g. clusters 8 and 14). In summary, we revealed extensive variation in the transcriptomic landscape of developing eye-antennal discs between D. melanogaster and D. mauritiana with different developmentally relevant processes being affected by these changes. 
bioRxiv preprint doi: https://doi.org/10.1101/2020.01.24.918011; this version posted June 14,2020 . The copyright holder for this preprint (which was not certified by peer review) is the author/funder, who has granted bioRxiv a license to display the preprint in perpetuity. It is made available under aCC-BY-NC-ND 4.0 International license.
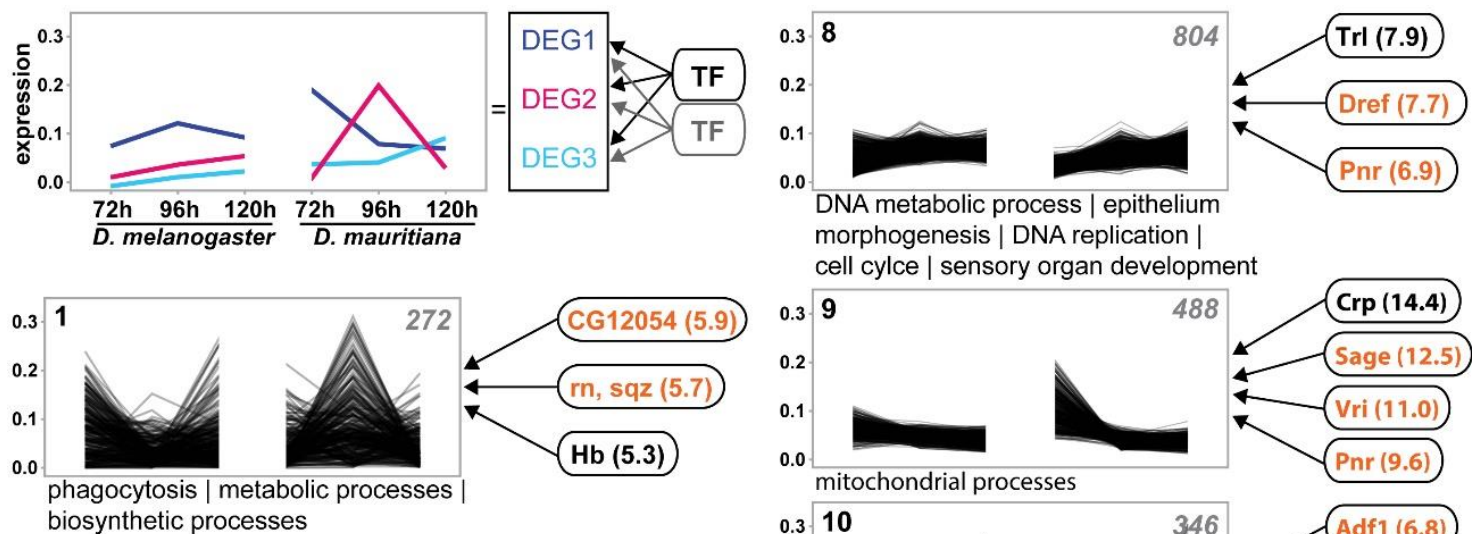

cell cylce | sensory organ development
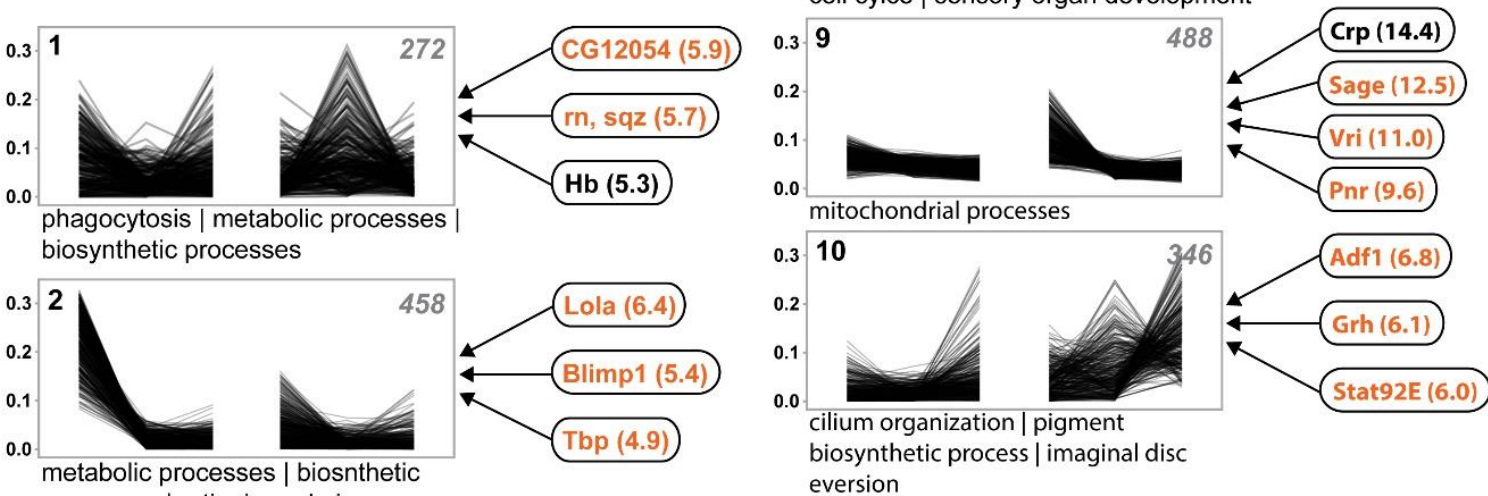

biosynthetic process | imaginal disc eversion
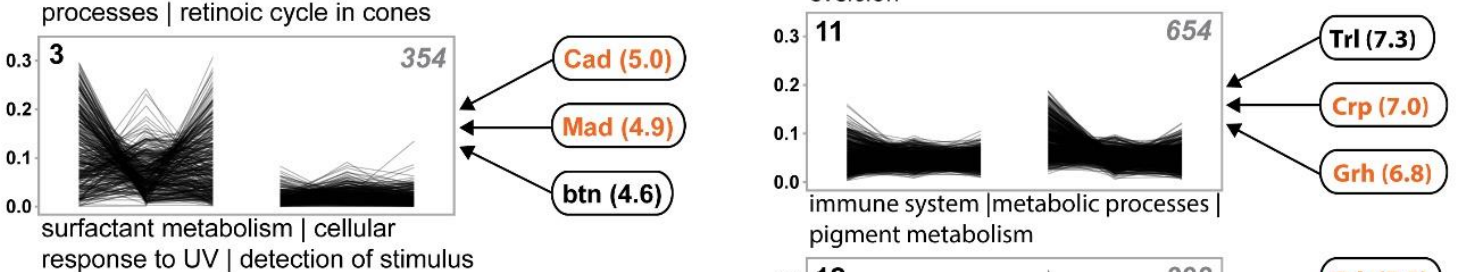

response to UV | detection of stimulus

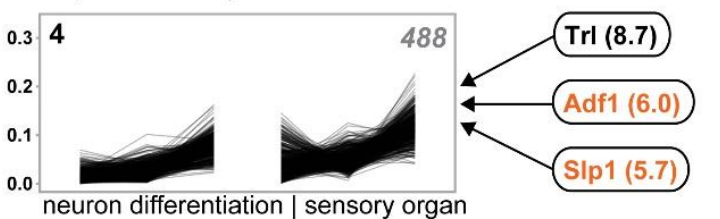

pigment metabolism

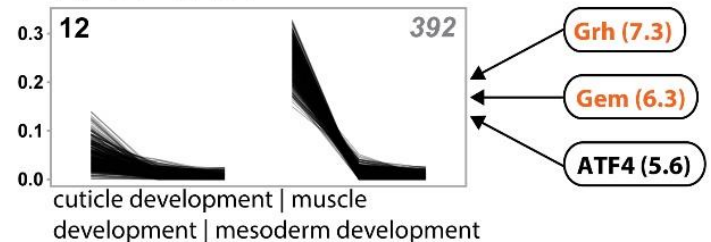

development | regulation of developmental processes | R7 cell differentiation
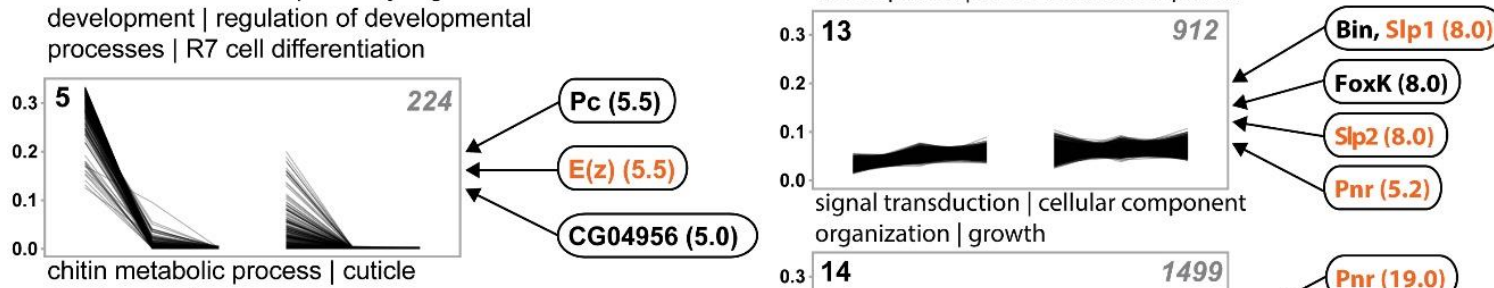

organization | growth
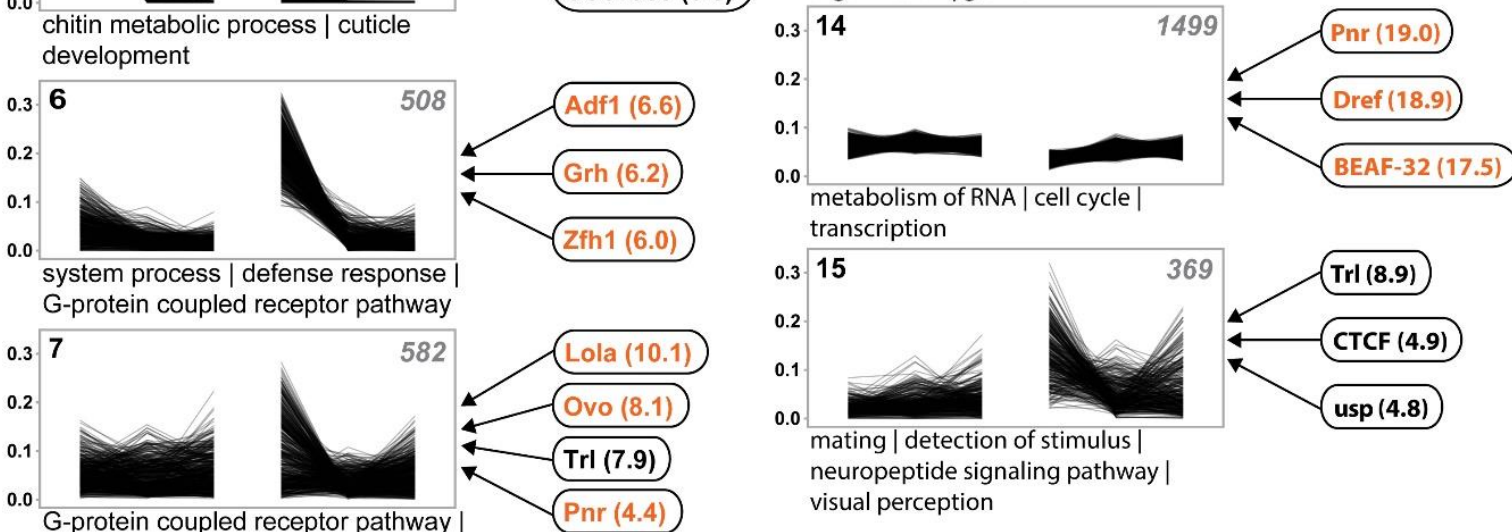

neuronal system | synaptic process

Fig 2. Extensive remodeling of the transcriptome underlying eye-antennal disc development. The scheme in the upper left corner illustrates the rational of the analysis. Differentially expressed genes (DEGs) with similar expression profiles were clustered and enriched transcription factor (TF) binding motifs were identified (see Materials and Methods for details). Cluster analysis of all differentially expressed genes between D. melanogaster and D. mauritiana. The number of genes in each cluster is given in the upper right corner. Significantly enriched GO terms are provided for each cluster. TFs refer to potential upstream regulators and the respective NES values (non-redundant occurrence and excluding non-Drosophila motifs). Factors written in orange are significantly differentially expressed in at least one stage between the two species. A complete list of enriched GOterms and potential upstream factors is available in S2 Table. 
Variation in the transcriptomics landscape is caused by variation in expression of hub genes

The interspecific differences in gene expression dynamics must be the result of variation in the underlying regulatory interactions. Hub genes occupy central positions in developmental GRNs affecting many target genes [29,30]. To identify putative hub genes that regulate the expression of DEGs, we assume that DEGs with similar expression profiles are regulated by similar transcription factors; i.e. we treated co-expression clusters (clusters in Fig 2) as "gene modules" [50-52]. We established stage-specific chromatin accessibility data (ATACseq) to identify enriched transcription factor binding motifs for each cluster (see Materials and Methods and S1 Fig for details). Each cluster showed enrichment of motifs for a unique combination of transcription factors (Fig 2), suggesting that variation in different gene modules is influenced by different putative hub genes.

Many hub genes play central roles during development $[31,32]$ and indeed, some of the identified transcription factors have previously been described to be involved in eye-antennal disc development. For instance, Lola (Fig 2; clusters 2, 5, 7 and 12) regulates ocelli [53] and photoreceptor and cone cell development [54], Blimp-1 (Fig 2; cluster 2) controls the progression of retinal differentiation [55,56] and Mad (Fig 2; clusters 3, 4 and 15), the transcription factor that mediates Decapentaplegic (Dpp) - signalling [57], is involved in various processes in the eye-antennal disc [58-60]. Additionally, in 5 of 15 clusters (NES > 4) (Fig 2; e.g. clusters 7-9, 13 and 14) (in 9 of 15 clusters, NES > 3) we found a strong enrichment of motifs predicted to be bound by the GATA transcription factor Pnr, which is involved in establishing the early dorsal ventral axis of the eye [61-63]. Therefore, we revealed putative hub genes with important roles during the eye-antennal disc development, which regulate DEGs between D. melanogaster and D. maurtiana. Interestingly, $61.3 \%$ (92 of 150) of the putative upstream transcription factors (i.e. putative hub genes) (NES > 4.0) were differentially expressed in at least one developmental stage (False discovery rate (FDR) 0.05) (Fig 2 and S2 Table). In summary, we identified many putative hub genes whose differential expression affected the transcriptomic landscape underlying eye-antennal disc development.

Pannier is a phenotypically relevant hub gene in the GRN underlying dorsal head development

The observation that many of the putative hub genes were differentially expressed between species suggests that these changes may be causal in defining the observed differences 
1 in eye size and head shape. However, developmental processes [64,65] tend to be robust against

2 perturbations because variation in the expression of developmental genes often does not result in phenotypic differences due to extensive buffering within GRNs [66-68]. Therefore, it remains questionable, whether the differential expression of the putative hub genes and thus the variation in expression of their target genes are indeed relevant for the differences in head morphology between D. melanogaster and D. mauritiana. To test this, we further investigated the role of Pannier (Pnr), which is an interesting putative hub gene for the following reasons: 1. Our global clustering and motif enrichment analyses suggest that Pnr regulates many DEGs between both species (Fig 2). 2. pnr itself is differentially expressed between D. melanogaster and D. mauritiana, with higher expression in the latter species (Fig 3A). 3. Pnr is known to be expressed in the dorsal eye-antennal disc [61] and it is involved in defining the dorsal-ventral axis of the eye $[61,63,69]$. Later during eye-antennal disc development, Pnr influences the ratio of retinal and head cuticle fate in the dorsal disc by repressing retinal determination genes $[61,70,71]$.

To confirm that $p n r$ is indeed a hub gene with many connections within the GRN orchestrating eye-antennal disc development, we first refined the list of its potential target genes. We searched for the Pnr-specific GATA motif in genomic regions that were accessible during eye-antennal imaginal disc development (see Materials and Methods and S1 Fig for details). In 14,511 open chromatin regions (across all timepoints) we found 1,335 Pnr-specific GATA motifs associated with 1,060 genes expressed in our RNAseq dataset, suggesting that those genes were active during eye and head development. In the regulatory regions of these 1,060 Pnr target genes we found a strong enrichment of Pnr binding sites (S4A Fig) and many of the genes were also predicted to be regulated by Pnr in our cluster analysis (see Fig 2 and S4B Fig). In line with the known functions of Pnr during eye-antennal disc development, the Pnr target genes were highly enriched in processes like compound eye development and growth as well as cell cycle progression (S4C Fig). Additionally, a cross-validation of our Pnr target genes with the DroID interaction database [72,73] showed that our list contained three known direct target genes (i.e. Pnr-regulatory sequence interaction; $d l, P c$ and $S f m b t$ ) and 23 genes with known genetic interactions (Fig 3B), suggesting that these genes are also direct targets of Pnr. 17 out of these 26 genes (65.38\%) (Fig 3B) and 716 of the full set of 1,060 target genes (67.5\%) showed expression differences between D. melanogaster and D. mauritiana (S3 Table). Taken together, we confirmed that Pnr regulates many DEGs during eye-antennal disc development, suggesting that it is a hub gene with phenotypic relevance. 
To test if variation in pnr expression can explain naturally occurring differences in eye size and dorsal head shape (Fig 1) we overexpressed and knocked down pnr in D. melanogaster

3 in the dorsal eye-antennal disc in a domain that is reminiscent of endogenous pnr expression $4 \quad$ (S5A and 5B Fig). Successful manipulation of the expression was confirmed by reduced and 5 enhanced protein expression in knock-down and overexpression discs, respectively (S5C-S5E

6 Fig). Subsequently, we quantitatively compared the adult head morphology applying geometric 7 morphometrics and ommatidia counting (see Materials and Methods for details). A principal 8 component analysis of head shape variation showed that principal component 2 (PC2) explained $19.2 \%$ of the observed variation in head shape and PC3 explained $6.7 \%$ (Fig 3C; note that PC1 captured a technical artefact, see S6 Fig). Variation along PC2 mainly captured differences in the proportion of eye $v s$. interstitial cuticle in the dorsal head, as well as the location of the ocellar region. Intriguingly, the overexpression of pnr in the dorsal head region resulted in a shift from a "D. melanogaster"-like shape towards a more "D. mauritiana"-like shape along PC2, with an enlargement of the eyes at the expense of a slight reduction of the interstitial cuticle (Fig 3C). Ommatidia counting in entire eyes confirmed that the increase in eye area upon pnr overexpression was indeed due to an increase in number of ommatidia (Fig 3D). 
bioRxiv preprint doi: https://doi.org/10.1101/2020.01.24.918011; this version posted June 14, 2020. The copyright holder for this preprint (which was not certified by peer review) is the author/funder, who has granted bioRxiv a license to display the preprint in perpetuity. It is made available under aCC-BY-NC-ND 4.0 International license.
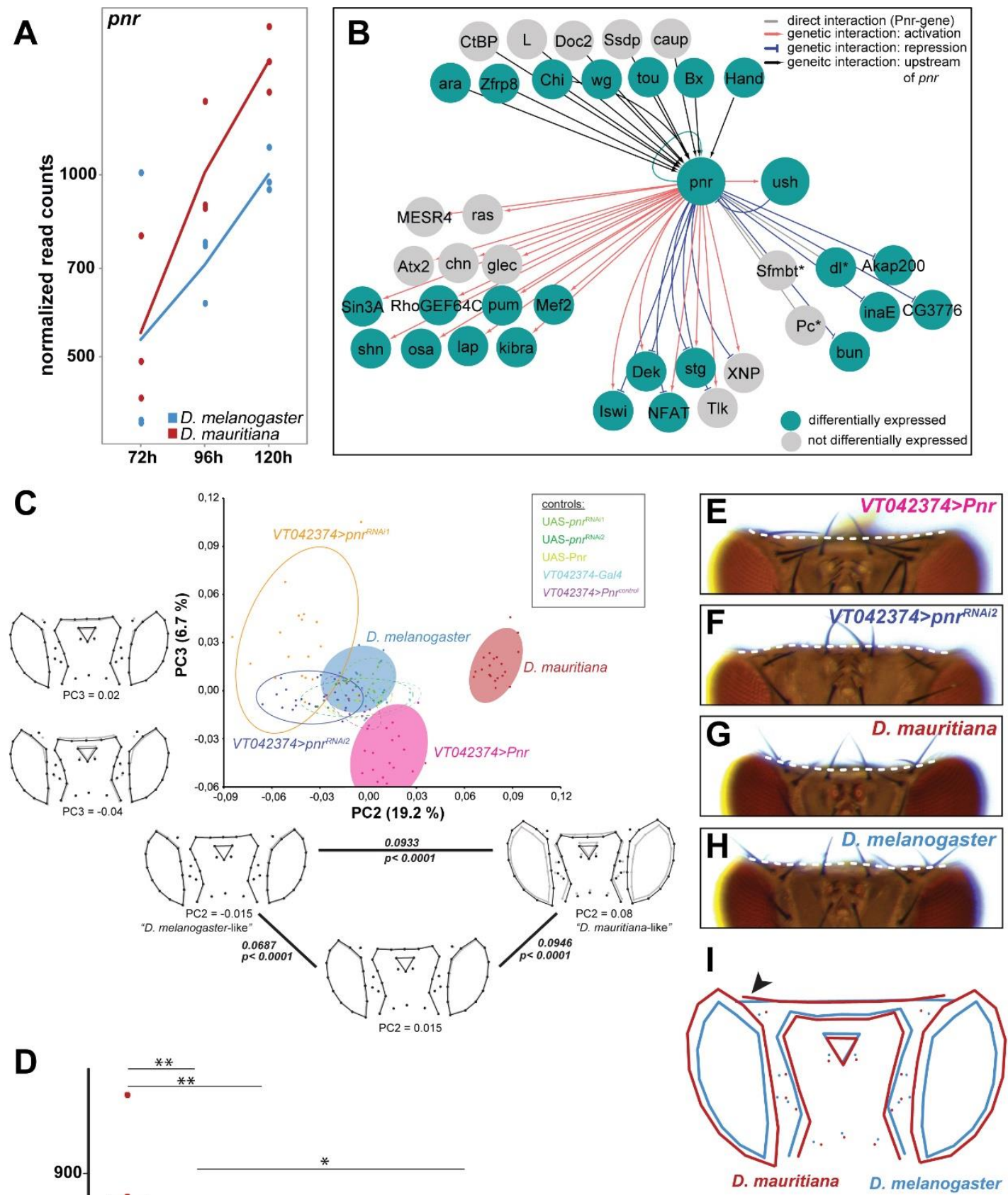

D
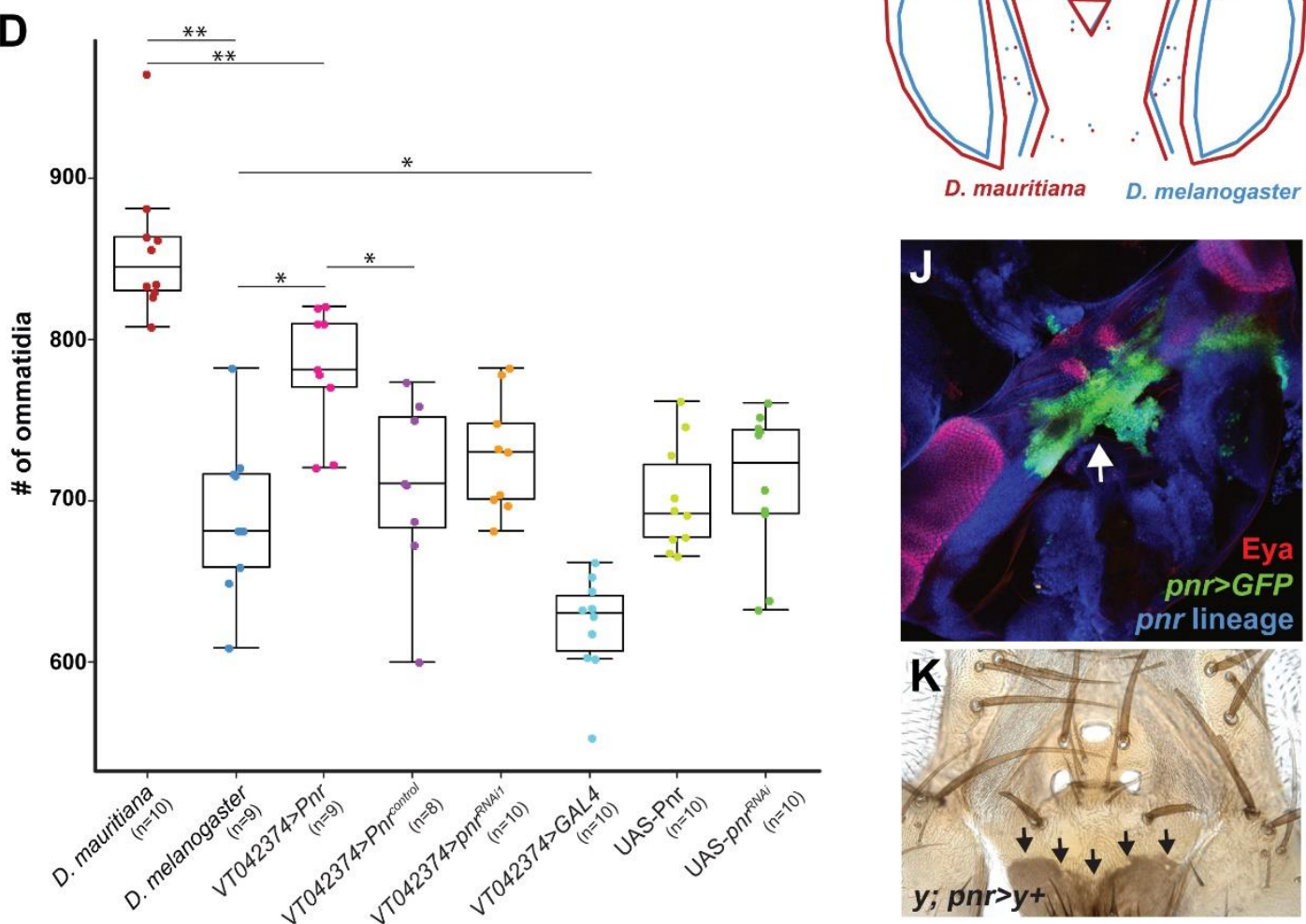
Fig 3. Pnr is a phenotypically relevant hub gene during eye-antennal disc development. (A) Expression dynamics of the $p n r$ transcript at the three developmental stages in D. melanogaster (blue) and D. mauritiana (red) based on rlog transformed read counts. (B) Network reconstruction of known interactions upstream and downstream of Pnr that overlap with our Pnr target gene list. 14 of the 29 target genes are activated (red edges) and 8 genes are repressed (blue edges) by Pnr. 6 target genes showed both types of interactions. 21 of the 30 putative Pnr target genes (68\%) were differentially expressed (green nodes). (C) Principal component analysis of dorsal head shapes. Shape outlines for PC values are given along the PC2 and PC3 axes, respectively, as indicated below each outline. In the triangle along PC2, the Procrustes distance between each shown shape outline is provided, as well as the p-value after permutation test with 1,000 cycles. Overexpression of pnr shifts the dorsal head shape towards a D. mauritiana head shape. (D) Number of ommatidia counted in one compound eye per individual. The

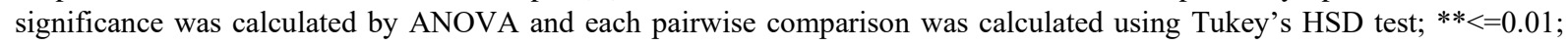
$*<=0.05$. The color code in $(\mathbf{C})$ and $(\mathbf{D})$ is the same. (E) Representative dorsal view of an adult head after overexpression of $p n r$ and (F) after knockdown of pnr. (G) Representative dorsal view of an adult head D. mauritiana. (H) Representative dorsal view of an adult head of D. melanogaster. The dotted lines in E-H mark the border of the occipital region. (I) Mean shapes of D. melanogaster (blue) and D. mauritiana (red) heads after discriminant function analysis including seven additional landmarks in the occipital region. The black arrowhead marks the lateral bending of the occipital region in D. mauritiana. (J) pnrexpression in developing pupal head structures. Cells marked with $p n r>$ GFP accumulated in the future occipital region (green) behind the developing ocelli (red), and the head region where the two discs fuse (white arrow). (K) pnr lineage in adult dorsal heads shown by cells in which yellow expression is restored (black arrows).

PC3 explained mostly differences in the dorsal-posterior head cuticle and the location of the ocellar region (Fig 3C). We analysed the dorsal-posterior head cuticle in more detail and observed that the occipital region was more convex upon pnr overexpression (Fig 3E), whereas downregulation consistently led to an enlargement of these regions (Fig 3F). Intriguingly, in accordance with a higher expression of pnr in D. mauritiana, we found a more convex occipital region in D. mauritiana (Fig 3G) compared to a more concave shape in D. melanogaster (Fig $3 \mathrm{H}$ and $3 \mathrm{I})$. Lineage tracing experiments showed consistent expression of pnr during eyeantennal disc development (S7 Fig) and detection of pnr expression in pupae (Fig 3J) as well as the analysis of pnr-expressing clones in adult heads (Fig 3K) confirmed that $p n r$ was indeed expressed in the future occipital region. In summary, we showed that Pnr is a phenotypically relevant hub gene during eye-antennal disc development because we were able to phenocopy aspects of the "D. mauritiana"-like head shape and eye size by upregulating pnr expression in the developing eye-antennal disc of D. melanogaster.

\section{U-shaped modulates Pnr function during eye-antennal disc development}

The detailed analysis of the heads of $p n r$ gain- and loss of function flies revealed a consistent gain- and loss of vertical bristles, respectively (Fig 4A-4C). A similar role in sensory bristle development has been shown in the wing imaginal disc, where Pnr controls the positioning sensory bristles in the thorax [74-76]. The role of Pnr in thoracic sensory bristle development is antagonized by the presence of its co-factor U-shaped (Ush) [74,77]. Upon heterodimerization with Ush, Pnr that normally acts as a transcriptional activator, acquires a repressive function [77-79]. In line with a dual role of Pnr as transcriptional activator and 
bioRxiv preprint doi: https://doi.org/10.1101/2020.01.24.918011; this version posted June 14, 2020. The copyright holder for this preprint (which was not certified by peer review) is the author/funder, who has granted bioRxiv a license to display the preprint in perpetuity. It is made available under aCC-BY-NC-ND 4.0 International license.

1 repressor, we found genes that were activated as well as repressed by Pnr among its

2 differentially expressed high confidence target genes during eye-antennal disc development (S8

$3 \quad$ Fig and Fig 3B). Additionally, the knock-down and overexpression of ush during eye-antennal

4 disc development resulted in loss or misplacement of vertical bristles (Fig 4D and 4E),

5 suggesting that Pnr and Ush may coordinate similar processes. Therefore, we asked whether

6 Ush may also modulate the function of Pnr during eye-antennal disc development. To answer

7 this question, we tested whether 1) Pnr and Ush are co-localized in the same cells in the eye-

8 antennal disc and 2) both genes interact genetically.
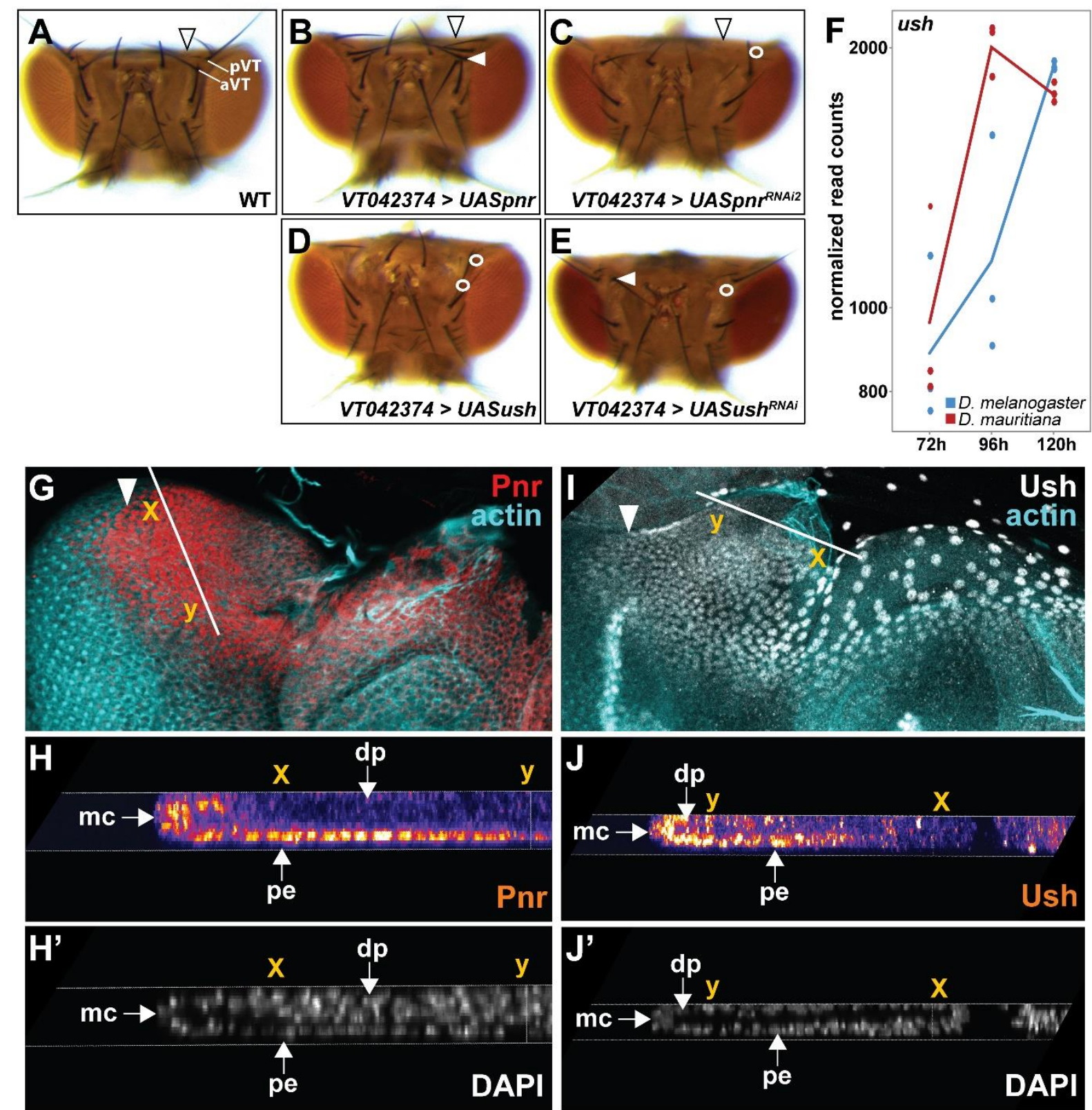

Fig 4. Pnr and Ush interact during eye-antennal disc development. (A) Dorsal view of an adult wild type head of $D$. melanogaster. Posterior (pVT) and anterior vertex bristles (aVT) are labelled. The arrowhead marks the occipital region. (B) Overexpression of pnr did not lead to major irregularities in the dorsal head cuticle, but to duplication of the pVT bristle next to the compound eye (white arrowhead) $(\mathrm{N}=9$ females, $\mathrm{N}=10$ males). (C) Knock-down of pnr resulted in visible enlargement 
of the occipital region (black arrowhead) and to the loss of the pVT bristle next to the compound eye (white circle) $(\mathrm{n}=10$ females, 10 males). (D) Overexpression of $u$ sh resulted in irregularities in the dorsal head cuticle, and to the loss of the pVT and aVT bristles (white circles) next to the compound eye ( $\mathrm{n}=10$ females). (E) Knock-down of ush resulted in slight irregularities of the dorsal head cuticle, to loss (white circle) or misplacement (white arrowhead) of the aVT bristle ( $\mathrm{n}=11$ females). (F) Expression dynamics of the ush transcript at the three developmental stages in D. melanogaster (blue) and $D$. mauritiana (red) based on rlog transformed read counts. $(\mathbf{G}, \mathbf{H})$ Pnr protein location in $3^{\text {rd }}$ instar eye-antennal discs in $D$. melanogaster. The Pnr protein is present in the dorsal peripodial epithelium (pe) of the developing disc (G), including a few cells of the margin cells (mc) and the disc proper (dp) (H'). The white arrow in $\mathbf{G}$ marks the morphogenetic furrow, the solid white line marks region of the cross section shown in $\mathbf{H}$ and $\mathbf{H}^{\prime}$ and the $\mathrm{x}$ and y coordinates indicate the same location in $\mathbf{G}, \mathbf{H}$ and $\mathbf{H}^{\prime}$. (I, J) Ush protein location in $3^{\text {rd }}$ instar eye-antennal discs in D. melanogaster. The Ush protein is, similarly to Pnr (compare to G), expressed in the dorsal peripodial epithelium (pe) of the developing disc (I), including a few cells of the margin cells (mc) and the disc proper (dp) (J'). The white arrow in I marks the morphogenetic furrow, the solid white line marks region of the cross section shown in $\mathbf{J}$ and $\mathbf{J}$ ' and the $\mathrm{x}$ and y coordinates indicate the same location in $\mathbf{I}, \mathbf{J}$ and $\mathbf{J}$. [61,78], we observed expression in all three studied stages in our RNAseq data (Fig 4F). As shown for pnr (Fig 3A), ush expression was mostly higher in D. mauritiana (Fig 4F). We confirmed the transcriptomics data using newly generated antibodies to show that both proteins are expressed in largely overlapping domains (Fig 4G-4J and S9 Fig) in the large nuclei of the dorsal peripodial epithelium (compare Fig 4G to 4I) and in a subset of the cuboidal margin cells in the disc proper (compare Fig $4 \mathrm{H}$ to $4 \mathrm{~J}$ ).

Since both proteins are co-localized in the eye-antennal disc, we tested for genetic interactions. We asked whether manipulation of pnr expression affected ush expression and vice versa. To this end, we overexpressed and knocked down both genes in the dorsal eyeantennal disc in a domain that is reminiscent of pnr expression (S5A and S5B Fig). We confirmed the successful overexpression and knock-down of both genes by enhanced and loss of protein expression, respectively (S5C-S5H Fig). Due to the qualitative nature of our experimental setup we could not obtain conclusive results for some of the tested combinations. However, we observed a reduced Ush signal upon knock-down of pnr (S5I Fig), suggesting a positive interaction between pnr and ush. Additionally, upregulation of $u s h$ resulted in slightly reduced Pnr protein levels (S5J Fig), suggesting a negative impact of Ush on pnr expression. Further support for this idea comes from the induction of a double-antenna phenotype when ush was overexpressed using a stronger pnr driver line (S5K Fig) [78], a phenotype that we also observed upon pnr knock-down (S5L Fig) [61]. In summary, we could show that Pnr and its co-factor Ush are co-expressed, interact genetically (S5M Fig) and are involved in dorsal sensory head bristle development. We suggest that a dual role of Pnr during eye-antennal disc development is most likely mediated by its co-factor Ush. 


\section{Discussion}

(

The trade-off between compound eyes and the interstitial cuticle in Drosophila heads is an excellent model to study the developmental and molecular mechanisms underlying complex trait evolution $[22-24,39,42,44]$. We established that this trade-off is present in the dorsal head of D. melanogaster and D. mauritiana, with the latter having larger compound eyes with significantly more ommatidia [this work and 42]. The comparison of larval eye-antennal disc development in both species revealed differences in the size of future head regions (i.e. retinal and antennal regions) starting at $96 \mathrm{~h} \mathrm{AEL,} \mathrm{suggesting} \mathrm{that} \mathrm{variation} \mathrm{in} \mathrm{processes} \mathrm{during} \mathrm{late}$ larval development are responsible for the observed differences in head morphology. To identify candidate genes driving these developmental differences, we combined comparative transcriptomics with chromatin accessibility data obtained prior to and after interspecific differences were observable (i.e. $72 \mathrm{~h}, 96 \mathrm{~h}$ and $120 \mathrm{~h}$ AEL). We show that the GATA transcription factor Pannier (Pnr) is a prime candidate hub gene: It showed higher expression in D. mauritiana and up to $67 \%$ of its more than 1,000 predicted direct target genes were differentially expressed between species. In the following we propose a developmental model explaining how variation in $p n r$ expression can simultaneously affect ommatidia number and interstitial cuticle size and we discuss our findings with respect to evolutionary hotspots and GRN evolution.

\section{Developmental mechanism underlying eye size and head shape differences between $D$. melanogaster and D. mauritiana}

The spatially restricted overexpression of $p n r$ in its endogenous domain resulted in a significant increase in ommatidia number, implying a direct effect on retinal development and thus final eye size. Our lineage tracing experiment showed that $p n r$ positive cells contribute to the entire dorsal peripodial epithelium and to cells of the dorsal posterior margin where the morphogenetic furrow, and thus retinal differentiation, is initiated at late second/early third instar stages [80]. It has recently been shown that Eyeless/Pax6 (Ey) activity in the peripodial epithelium and in these posterior margin cells is necessary for morphogenetic furrow initiation and placement of the dorsal-ventral boundary [81]. The latter is tightly linked to reported functions of Pnr and tissue growth in the retinal region of the eye-antennal disc [61,63,69]. We found ey among the putative direct Pnr target genes, suggesting that Pnr may activate ey expression in the peripodial epithelium and in posterior margin cells. Since ey was not 
1 differentially expressed in our dataset, it remains to be tested if Pnr may regulate the final number of ommatidia clusters and thus final eye size through Ey-mediated initiation of differentiation and regulation of overall retinal growth prior to the stages studied here (Fig 5A).

We found that pnr-expressing cells contribute to the dorsal interstitial head region in pupae, suggesting that the phenotypic effects in the occipital head region most likely highlight late functions of Pnr. Indeed, Pnr represses members of the retinal determination network throughout the third instar stage to regulate the ratio of retinal cells $v s$. head cuticle cells [71]. In addition to ey, we found eyegone (eyg) as second member of the retinal determination network among putative direct Pnr targets. Both genes must be repressed to ensure proper dorsal interstitial cuticle development [82,83], suggesting that Pnr may act through transcriptional repression during late dorsal head development. Such a repressive role could be mediated upon heterodimerization with its co-factor Ush as shown for the wing disc $[70,77,84,85]$. Indeed we observed that, despite previous reports [61], the Ush protein is co-localized with Pnr in dorsal peripodial epithelium and in the cuboidal cells of the disc margin. In accordance with their coexpression, both genes interact genetically and they are both necessary for proper dorsal head development and the formation of the same head bristles (this work and see [74] for bristle phenotype). Therefore, we propose that Ush mediates a repressive late function of Pnr in the dorsal peripodial epithelium to regulate retinal $v s$. dorsal head cuticle development during the third larval instar stage (Fig 5A).

Taken together, our data shows that variation in $p n r$ expression during late larval development can simultaneously affect the size of the dorsal interstitial head cuticle and the compound eyes (Fig 5A). Accordingly, increasing pnr expression levels in D. melanogaster mimics higher pnr expression as observed in D. mauritiana and phenocopied major aspects of the dorsal D. mauritiana head shape and eye size. Consistent with the fact that variation in complex traits is caused by multiple loci $[15,17]$, pnr overexpression was not sufficient to fully phenocopy D. mauritiana head shape and eye size. Furthermore, it remains to be established whether the $p n r$ and/or $u s h$ loci contain genetic variants associated with eye size and head shape differences. While quantitative genetics approaches are not applicable due to the infertility of F1 females in interspecific crosses between D. melanogaster and D. mauritiana [86], reciprocal hemizygosity tests [87] for Pnr, Ush and putative regulators of these two factors represent a powerful tool to further dissect the causative genetic variants in the future.

In summary, based on our developmental model the increase in eye size in D. mauritiana that goes along with a reduction of dorsal interstitial cuticle and a convex bending of the 
1 occipital head region can partially be explained by natural variation in $p n r$ expression. Since

2 Pnr is a highly connected pleiotropic transcription factor that regulates many differentially expressed genes between D. melanogaster and D. mauritiana, we argue that Pnr is a phenotypically relevant hub gene underlying natural variation in head morphology.

Functional constraints drive co-evolution of phr and $u s h$ expression

In line with the pleiotropic role of Pnr during eye-antennal disc development, classical gain- and loss of function experiments resulted in extensive phenotypic consequences ranging from retinal overgrowth to the entire loss of the compound eyes $[61,71]$. These rather coarse experiments based on highly artificial systems, such as ectopic expression of pnr induced by an eyeless/Pax6-Gal4 driver line that is active from early stages on in most of the eye-antennal disc [81], are suitable to unravel the combined functions of a pleiotropic gene. However, it is a major challenge to disentangle the distinct tissue and stage specific functions of pleiotropic factors and find out which of these are phenotypically relevant in an evolutionary context $[35,88]$. This is particularly relevant because developmental processes [64,65] and the expression of the genes regulating them [89-91] tend to be robust against genetic or environmental perturbations. Therefore, functional and developmental constraints limit the evolutionarily relevant phenotypic effects of pleiotropic genes (Fig 5B).

We observed an excellent example for such a constraint. While individual gain- and loss of function of pnr and ush in D. melanogaster affected the formation of anterior and posterior vertical sensory bristles in the dorsal head, we never observed gain or loss of these bristles between species. Pnr and Ush have been shown to be involved in thoracic sensory bristle development by controlling the positioning of proneural cell clusters in the wing disc [74-76]. Thoracic sensory bristles develop in regions with high pnr and low ush expression [74,77] and their balanced expression is facilitated by genetic interactions between both genes [84]. We observed that pnr overexpression in the eye-antennal disc consistently resulted in duplication of the anterior vertical bristles, suggesting that extra bristles arise where $p n r$ overexpression cannot be compensated by Ush [see also 76]. Similarly, overexpression of $u s h$ in most of the dorsal peripodial epithelium resulted in loss of the anterior and posterior vertical bristles, showing that extra Ush above a certain threshold completely antagonizes Pnr function and subsequent sensory bristle formation. Hence, the stoichiometry between Pnr and its co-factor Ush is crucial for proper sensory bristle development in the dorsal head as well. 
How can natural variation in $p n r$ and $u s h$ expression cause differences in head morphology without affecting the sensory bristle pattern? Since most of the proneural cell clusters are specified $18 \mathrm{~h}$ to $6 \mathrm{~h}$ prior to puparium formation [92], the ratio between Pnr and Ush must remain stable at $72 \mathrm{~h}$ and $96 \mathrm{~h}$ AEL to ensure proper sensory bristle formation. However, once bristles are specified, variation in the ratio of both factors at later stages (e.g. $120 \mathrm{~h} \mathrm{AEL)}$ can cause natural variation in the shape of the dorsal occipital head region without affecting bristle formation (Fig 5C). Accordingly, both genes showed higher expression in D. mauritiana at $72 \mathrm{~h}$ and $96 \mathrm{~h} \mathrm{AEL} \mathrm{resulting} \mathrm{in} \mathrm{a} \mathrm{similar} \mathrm{ratio} \mathrm{of} \mathrm{both} \mathrm{factors} \mathrm{(Fig} \mathrm{5C).} \mathrm{In} \mathrm{contrast,} \mathrm{a} \mathrm{different}$ ratio was observed at $120 \mathrm{~h}$ AEL, with pnr expression being higher in D. mauritiana, while ush expression was more similar in both species at this later stage (Fig 5C). We propose that the functional constraint on stereotypic sensory bristle development drove the co-evolution of pnr and ush expression during early third instar disc development in D. melanogaster and D. mauritiana (Fig 5C). Indeed, according to their crucial function in environmental perception [93], vertical bristles have been shown to be robust against temperature fluctuations during development [94]. Therefore, the context-dependent presence and action of a transcriptional co-factor provides a versatile mechanism to restrict the phenotypic effects of a pleiotropic developmental factor in an evolutionary context (Fig 5B).

\section{Repeated evolution of hotspot genes may play a minor role for complex trait evolution}

Natural intra- and interspecific variation in eye size and a trade-off between retinal tissue and head cuticle is a common feature of Drosophila [22,24,39,41,42,44,48]. The most comprehensive genetic and developmental characterization of this trait suggested that differences in the early subdivision of the eye-antennal disc driven by variation in the temporal regulation of eyeless/Pax6 expression is a common mechanism driving the trade-off [44]. In line with the identification of evolutionary hotspot genes for rather simple traits, such as variation in pigmentation patterns [10,11] and the gain and loss of structures [12-14], this finding suggests that repeated natural variation in hotspot genes may be a major driver for the evolution of complex morphological traits as well [44]. In contrast to these results, our comparison of eye-antennal disc development between D. melanogaster and D. mauritiana showed that differences in the anlagen of the future retinal field and the antenna arise only late during the third instar stages. Accordingly, our developmental model involving natural variation in pnr and ush expression (Fig 5A) provides an alternative developmental mechanism 
that can explain the simultaneous effects on ommatidia number variation and differences in the interstitial head cuticle.

Recent advances in studying the developmental and genetic basis of the trade-off between eye and interstitial cuticle size in Drosophila revealed that differences in developmental processes affecting both traits simultaneously seem to be common in Drosophila [23,24]. However, eye and interstitial cuticle size can also be genetically and developmentally uncoupled as shown by a detailed characterization of the head trade-off in D. melanogaster and D. simulans [22]. Quantitative genetics data for intraspecific variation in the head trade-off in D. melanogaster and D. simulans shows that its genetic basis is highly diverse [23], supporting our observation that repeated evolution through hotspot genes may not be prevalent in the evolution of head morphology.

The insect compound eye is composed of hundreds of functional subunits, the ommatidia, and they themselves are built by 14 unique cells [95]. Therefore, the size of an insect compound eye can vary due to the number of ommatidia or due to their size. Indeed, a detailed morphological analysis showed that differences in the number of ommatidia explains intra- and interspecific variation in various Drosophila species [this work and 23,42,44], while eye size differences between $D$. simulans and D. mauritiana are caused by variation in ommatidia size [22,42]. Since these two features are regulated at different time-points by different developmental processes, it is expected that the molecular and developmental basis of eye size differences varies in different groups. Therefore, depending on the cellular basis of observed morphological differences, different developmental and molecular mechanisms can affect complex trait evolution.

A potential explanation for the convergent evolution of the head trade-off may be the complex modular nature of head development. The eye-antennal disc comprises compartments for all future head structures, including the eye, the interstitial cuticle, and the antennae. Besides the already established mechanisms [this work and 44], it is conceivable that differences in adult head morphology can be the result of variation in different developmental processes in each compartment of the disc at different time points. Additionally, the development of a complex organ, such as the insect compound eye, is controlled by several hub genes. This is best exemplified by the observation that the experimental mis-expression of different members of the retinal determination network can induce an ectopic retinal developmental program [96100], suggesting that they all act as input-output devices in the retinal GRN. In contrast, the development of rather simple morphological traits is regulated by very few hub genes that act 
1 as input-output devices integrating positional information and activating most target genes

2 required to initiate the entire developmental program to form the structure $[33,34]$. In summary,

3 our current knowledge based on quantitative genetics, developmental as well as morphological

4 data suggests that different nodes within the GRN underlying head and eye development evolve

5 to give rise to variation in head morphology in Drosophila. We argue that the complexity and

6 modularity of complex organ development facilitates the convergent evolution of complex

7 morphological traits. 
bioRxiv preprint doi: https://doi.org/10.1101/2020.01.24.918011; this version posted June 14, 2020. The copyright holder for this preprint (which was not certified by peer review) is the author/funder, who has granted bioRxiv a license to display the preprint in perpetuity. It is made available under aCC-BY-NC-ND 4.0 International license.
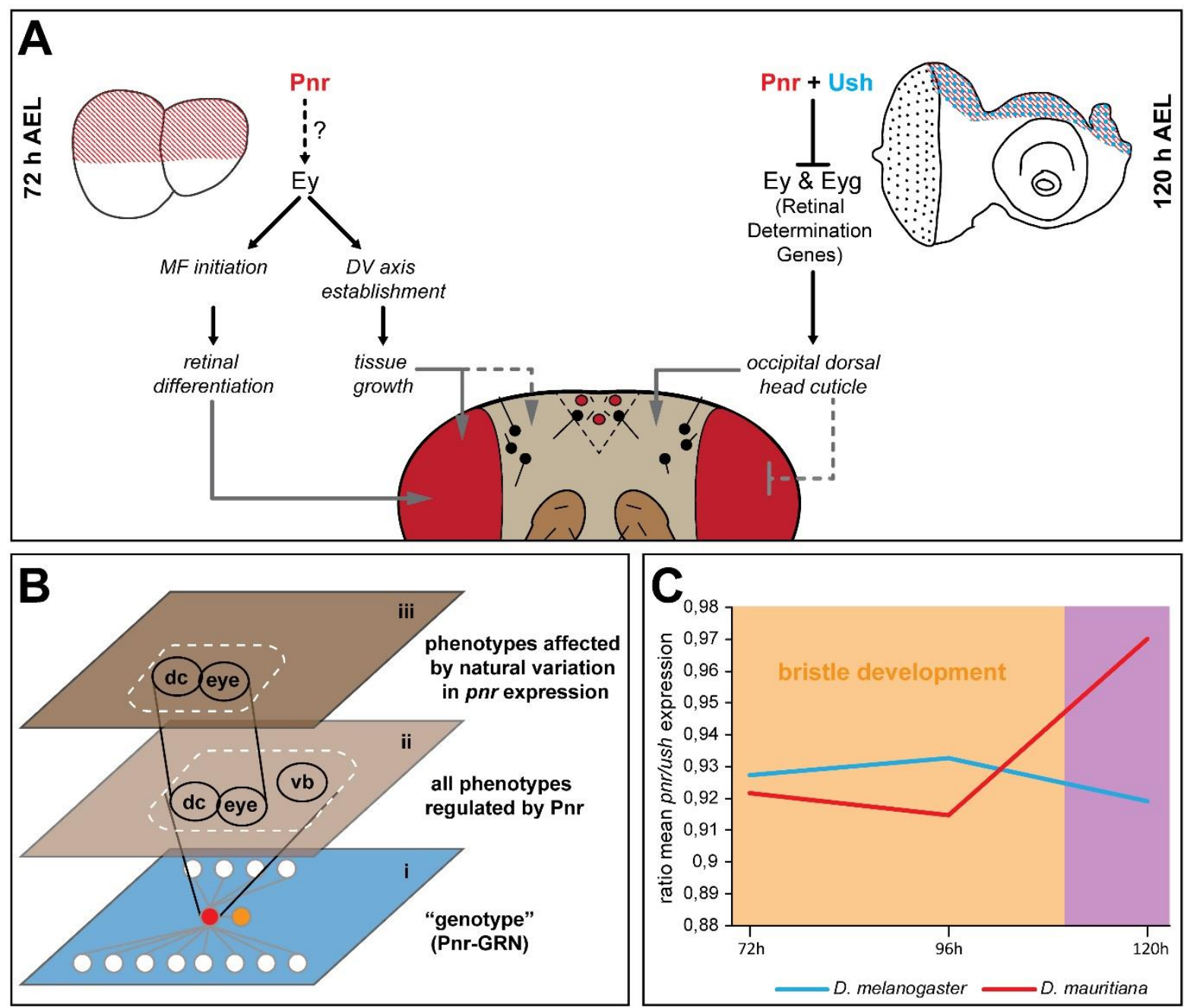

D

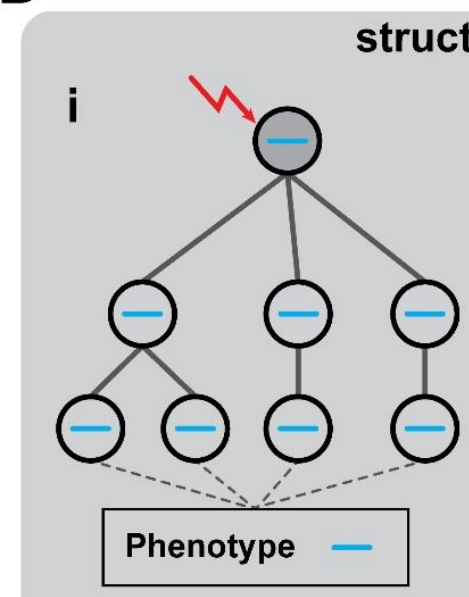

buffering at gene locus
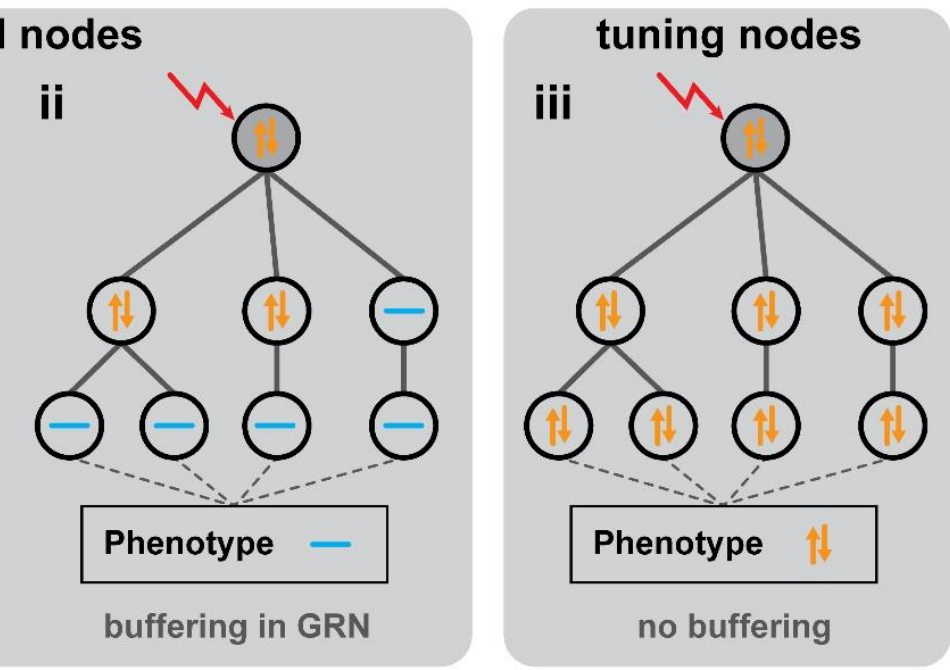

gene affected by mutation

gene expression stable

gene expression changed

Fig 5. Developmental model and hypothesis about GRN evolution. (A) Summary of expression and functional data obtained in this work. Black connections indicate genetic interactions among genes. Grey connections represent observed phenotypic effects upon manipulation of gene expression. (B) Schematic representation of functional constraints on vertical bristle development. The genotype level (i) is depicted as GRN centred on Pnr (red node in i). Pnr regulates the development of the 
dorsal occipital cuticle (dc), the compound eye (eye) and vertical bristles (vb) (ii). Due to a functional constraint on vertical bristle development only the effect of differences of $p n r$ expression on dc and eye are phenotypically relevant during the evolution of head morphology (iii). We propose that the constraint effect of $p n r$ expression differences between $D$. melanogaster and D. mauritiana is mediated by co-evolution of ush expression (orange node in i). (C) The ratio of pnr and ush expression is stable at $72 \mathrm{~h}$ and $96 \mathrm{~h}$ AEL when vertical bristles are defined. Later expression of both genes is not constraint anymore by a function in bristle development. (D) Schematic representation of structural (i, ii) and tuning nodes (iii) in developmental GRNs.

\section{Context dependent regulatory interactions facilitate the evolution of pleiotropic hub} genes

Although we did not find indications for repeated evolution at hotspot genes for natural variation in eye size and head shape in Drosophila, our data and the work of others consistently revealed variation in the expression and function of crucial pleiotropic genes contributing to morphological evolution [e.g. this work and 6,7,44]. On the first glance this observation is counter-intuitive for two reasons: 1) The mutation rates of central developmental regulators, such as transcription factors and signalling molecules, are low [101-103] and such factors tend to be functionally conserved across the animal kingdom [97]. 2) Systematic analyses of GRN connectivity showed that developmental regulators are often highly connected $[104,105]$. Since GRNs are thought to be scale-free with few highly connected hub genes, mutations are more likely to occur in the many weakly connected peripheral genes [15,30]. Therefore, the question arises, why morphological evolution is often driven by natural variation in expression of highly connected pleiotropic genes?

First, although a high level of network connectivity ensures network stability, data obtained in yeast showed that genes that are regulated by many other factors (trans-mutational target size) and genes that contain many transcription factor binding motifs (cis-mutational target size) are more prone to accumulate mutations [90,106]. A study of Mendelian and complex disease genes revealed that they are often highly connected and that they act as brokers, connecting genes that are themselves poorly connected. Such broker positions could represent fragile nodes and variation in such genes may more likely result in phenotypic differences [107]. Although it remains to be tested if highly connected developmental regulators occupy similar fragile positions within GRNs, the position of pleiotropic genes within GRNs may influence the evolvability of their expression or function.

Second, highly connected genes often contain a complex regulatory landscape with many cis-regulatory elements allowing for their incorporation in different developmental GRNs. Consequently, a complex gene regulation facilitates the remodeling of regulatory interactions in a temporally and spatially defined manner [reviewed in 108]. Eye-antennal disc 
1 development is highly complex and the regulatory interactions within the underlying GRN are

2 variable both throughout time [49] and in different parts of the disc [37], requiring the use of the same developmental gene products in different contexts. For instance, genes of the retinal determination network are essential for the initial proliferation and growth of the entire eyeantennal disc $[56,109,110]$ and later they play a pivotal role in retinal specification $[80,111]$. These distinct roles have been suggested to be achieved by considerable rewiring of the respective GRNs, which allows them to fulfil temporally and even spatially restricted tasks [112]. The various described roles for Pnr during eye development [61,71], its continuous expression in the dorsal eye-antennal disc and the observation that variation in $p n r$ expression affects overall head shape and eye size simultaneously (this work), strongly suggest that Pnr as well is involved in several sub-networks during eye and head development. The interaction with co-factors, such as Ush provides a mechanism of modulating the role of Pnr from an activating to a repressing transcription factor and its usage in spatially defined GRNs. Hence, the contextspecific modulation of expression of a highly pleiotropic developmental factor, such as pnr and its target genes is facilitated by complex and modular gene regulation mechanisms and a highly dynamic underlying GRN.

\section{A versatile method to identify tuning nodes within developmental GRNs underlying morphological evolution}

Since natural variation in gene expression is a major driver of morphological divergence [33,113], many studies aiming at revealing candidate genes underlying the evolution of morphological traits employ comparative transcriptomics methods to study differential gene expression. However, changes in gene function and expression are often buffered in GRNs to maintain a stable phenotypic outcome [68,114-119]. This is best exemplified by the observation that the individual loss of function of many genes in yeast [116] and the nematode Caenorhabditis elegans [118] rarely has deleterious effects for the organism. In accordance with a generally conserved adult head morphology in D. melanogaster and D. mauritiana, we found that the expression of many genes representing key developmental and metabolic processes was conserved between the two species. However, the observed quantitative interspecific differences in eye size and head shape must be due to variation in some parts of the GRN which are then selected for if it translates into phenotypic diversity that is advantageous for the organism [120-122]. 
In the light of the observed discrepancy between a generally high network stability and the flexibility in some parts of the network, we propose that developmental GRNs must contain at least two types of nodes (i.e. genes): structural nodes and tuning nodes (Fig 5D). Structural nodes confer phenotypic stability because variation in such nodes (e.g. variation in expression of the gene) is either prevented by tight regulation at the locus (Fig 5Di) or buffered downstream in the GRN (Fig 5Dii). Such a robustness has been demonstrated to be often the result of redundancies [114], either on the level of cis-regulatory elements [115,119] or transcription factors and signaling molecules [68,117]. In contrast, tuning nodes represent genes for which variation is tolerated within the GRN and results in quantitative phenotypic variation (Fig 5Diii). Natural variation in ey [44] and pnr expression (this work), which translates into head shape and eye size differences, as well as the previously identified hotspot genes underlying variation in trichome formation [12] and body pigmentation [10,11] are excellent examples of such tuning nodes. It is important to note here that developmental genes may represent tuning nodes in some contexts, while they are structural nodes in most other contexts. Our observation that interspecific differences in pnr expression affected the dorsal head shape and eye size, but not sensory bristle formation is an excellent example for the context-dependence of tuning and structural nodes. Revealing structural and tuning nodes in developmental GRNs allows gaining new insights into constrained and variable developmental processes, respectively.

Since many highly connected hub genes with pleiotropic functions have been shown to regulate morphological differences, they can be identified based on their effect on their target genes [36]. A systematic analysis of GRN properties suggests that transcription factor-to-gene interactions are scale-free, meaning that some transcription factors regulate a high number of genes [30]. Therefore, we hypothesized that putative tuning nodes (e.g. genes coding for transcription factors) can be elucidated by their impact on the expression of their target genes [i.e. "gene modules"; 50-52]. Accordingly, our methodological framework combines the identification of DEGs, clustering of DEGs based on their developmental expression dynamics and the identification of putative shared upstream regulators integrating genome wide chromatin accessibility data (Fig 2). Since fluctuations in gene expression are often buffered within GRNs, not all expression differences of putative tuning nodes will result in phenotypic differences. Therefore, a functional validation is crucial to reveal phenotypically relevant tuning nodes. Such a validation can be achieved by applying classical developmental genetics methods as shown in this work. If such tools are not established, a tuning node could be validated using a hemizygosity test based on widely applicable CRISPR/Cas9 aided genome editing [87]. 
Our approach unfolds its full potential if complemented with quantitative genetics data

2 to identify exact genetic variants constituting the tuning node. This is relevant because mutations affecting the expression or function of tuning nodes could be in its locus itself or it could be the result of genetic changes in an upstream regulator. In the latter case it is expected that the number of potential candidates upstream of the tuning node is low because systematic analyses of GRN architecture revealed that the in-degree within networks follows a random distribution, suggesting that most transcription factors tend to be regulated by only a few upstream factors [30]. Therefore, once putative tuning nodes are identified by comparative transcriptomics, the number of candidate genes that regulate the tuning node is expected to be low facilitating further molecular characterization.

In summary, our comparative transcriptomics approach can be used as entry point to study the evolution of complex morphological traits or it can be applied to link already identified genetic variation to nodes within developmental GRNs and to developmental processes. Since the individual steps are applicable in many organisms, including those for which quantitative genetics approaches are not applicable, we are convinced that the approach represents a versatile framework to study the molecular and developmental basis of morphological evolution.

\section{Material and Methods}

A detailed description of all methods is available as Extended Materials and Methods in the Supporting Information.

\section{Comparative Transcriptomics and ATACseq}

Eye-antennal imaginal discs were dissected $72 \mathrm{~h}, 96 \mathrm{~h}$ and $120 \mathrm{~h}$ after egg laying (AEL) from larvae of D. melanogaster (Oregon R) and D. mauritiana (TAM16), respectively. Total RNA was extracted from the discs and libraries for Illumina HiSeq2000 sequencing were generated using the TruSeq RNA Sample Preparation Kit (Illumina, catalog ID RS-122-2002). Reads were mapped using Bowtie2 v. 2.3.4.1 [123], mapping data was processed using samtools version 1.9 [124,125] and differential expression analysis was performed with the DESeq2 package (DESeq2_1.22.2 [126]; R version 3.5.2). Metascape was used to analyse differential enrichment of GO terms for pairwise comparisons. Clustering of genes according to their developmental expression dynamics was performed with the coseq package (version 
1.6.1) $[127,128]$ and we searched for potential upstream factors using the i-cisTarget tool $[129,130]$. ATACseq to assess chromatin accessibility was perfromed as described before [131]. The pipeline used to define a high confidence target gene list of Pnr is described in detail in the Extended Materials and Methods.

\section{Genetic crosses, Immunohistology and phenotyping}

All fly crosses to knock-down and overexpress $p n r$ and $u s h$, as well as crosses for lineage tracing experiments were performed at $25^{\circ} \mathrm{C}$ and at a constant $12 \mathrm{~h}: 12 \mathrm{~h}$ light:dark cycle. We generated polyclonal antibodies against Pnr [132] and Ush [78] based on previous knowledge (Proteintech, Rosemont, IL, USA) and immunohistology was performed as described in the Extended Materials and Methods. Adult heads were either mounted in Hoyer's medium:Lactic Acid (50:50) or directly imaged for ommatidia counting or shape analysis using Geometric Morphometrics.

\section{Funding}

NP and EB are funded by the Deutsche Forschungsgesellschaft (DFG, Grant Number: PO 1648/3-1) to NP. FC acknowledges funding through grants BFU2015-66040-P, PGC2018093704-B-I00 and MDM-2016-0687, from Ministerio de Ciencia, Innovación y Universidades of Spain.

\section{Acknowledgments}

Many thanks to Marita Büscher and Kolja Eckermann for sharing antibodies and to Alistair P. McGregor, Marc Haenlin and Gerd Vorbrüggen for sharing Drosophila stocks. We are grateful for financial support for the ATACseq data, as well as for critical comments on an earlier version of this manuscript to Alistair P. McGregor. Stocks obtained from the Bloomington Drosophila Stock Center (NIH P40OD018537) were used in this study. Many thanks to the Deep-Sequencing Core Facility of the Universitätsmedizin Göttingen (UMG) for next generation sequencing. We are grateful for many fruitful discussions and feedback from members of the Department of Developmental Biology and Posnien Lab members.

\section{References}

1. Alberch P. From genes to phenotype: dynamical systems and evolvability. Genetica. 1991; 84: 511. doi: 10.1007/bf00123979.

2. Lewontin RC. The genetic basis of evolutionary change. New York: Columbia Univ. Pr; 1974.

3. Jeong S, Rokas A, Carroll SB. Regulation of body pigmentation by the Abdominal-B Hox protein and its gain and loss in Drosophila evolution. Cell. 2006; 125: 1387-1399. doi: 10.1016/j.cell.2006.04.043. 
bioRxiv preprint doi: https://doi.org/10.1101/2020.01.24.918011; this version posted June 14,2020 . The copyright holder for this preprint (which was not certified by peer review) is the author/funder, who has granted bioRxiv a license to display the preprint in perpetuity. It is made available under aCC-BY-NC-ND 4.0 International license.

4. Rebeiz M, POOL JE, Kassner Va, AQUADRO CF, Carroll SB. Stepwise modification of a modular enhancer underlies adaptation in a Drosophila population. Science. 2009; 326: 1663 1667. doi: $10.1126 /$ science. 1178357.

5. Hoekstra HE, Hirschmann RJ, Bundey RA, Insel PA, Crossland JP. A single amino acid mutation contributes to adaptive beach mouse color pattern. Science. 2006; 313: 101-104. doi: 10.1126/science.1126121.

6. McGregor AP, Orgogozo V, Delon I, Zanet J, Srinivasan DG, Payre F, et al. Morphological evolution through multiple cis-regulatory mutations at a single gene. Nature. 2007; 448: $587-$ 590. doi: 10.1038/nature05988.

7. Shapiro MD, Marks ME, Peichel CL, Blackman BK, Nereng KS, Jónsson B, et al. Genetic and developmental basis of evolutionary pelvic reduction in threespine sticklebacks. Nature. 2004; 428: 717-723. doi: 10.1038/nature02415.

8. Menuet A, Alunni A, Joly J-S, Jeffery WR, Rétaux S. Expanded expression of Sonic Hedgehog in Astyanax cavefish. Multiple consequences on forebrain development and evolution. Development. 2007; 134: 845-855. doi: 10.1242/dev.02780.

9. Stemmer M, Schuhmacher L-N, Foulkes NS, Bertolucci C, Wittbrodt J. Cavefish eye loss in response to an early block in retinal differentiation progression. Development. 2015; 142: $743-$ 752. doi: 10.1242/dev.114629.

10. Gompel N, Carroll SB. Genetic mechanisms and constraints governing the evolution of correlated traits in drosophilid flies. Nature. 2003; 424: 931-935. doi: 10.1038/nature01787.

11. Prud'homme B, Gompel N, Rokas A, Kassner Va, Williams TM, Yeh S-D, et al. Repeated morphological evolution through cis-regulatory changes in a pleiotropic gene. Nature. 2006; 440: 1050-1053. doi: 10.1038/nature04597.

12. Sucena E, Delon I, Jones I, Payre F, Stern DL. Regulatory evolution of shavenbaby/ovo underlies multiple cases of morphological parallelism. Nature. 2003; 424: 935-938. doi: 10.1038/nature01768.

13. Chan YF, Marks ME, Jones FC, Villarreal G, Shapiro MD, Brady SD, et al. Adaptive evolution of pelvic reduction in sticklebacks by recurrent deletion of a Pitx1 enhancer. Science. 2010; 327: 302-305. doi: 10.1126/science.1182213.

14. Shapiro MD, Bell MA, Kingsley DM. Parallel genetic origins of pelvic reduction in vertebrates. Proceedings of the National Academy of Sciences of the United States of America. 2006; 103: 13753-13758. doi: 10.1073/pnas.0604706103.

15. Boyle EA, Li YI, Pritchard JK. An Expanded View of Complex Traits: From Polygenic to Omnigenic. Cell. 2017; 169: 1177-1186. doi: 10.1016/j.cell.2017.05.038.

16. Mackay TF. Quantitative trait loci in Drosophila. Nature Reviews Genetics. 2001; 2: 11-20. doi: $10.1038 / 35047544$.

17. Mackay TFC, Stone Ea, Ayroles JF. The genetics of quantitative traits. Challenges and prospects. Nature Reviews Genetics. 2009; 10: 565-577. doi: 10.1038/nrg2612.

18. Burgio G, Baylac M, Heyer E, Montagutelli X. Genetic analysis of skull shape variation and morphological integration in the mouse using interspecific recombinant congenic strains between C57BL/6 and mice of the mus spretus species. Evolution. 2009; 63: 2668-2686. doi: 10.1111/j.1558-5646.2009.00737.x.

19. Maga AM, Navarro N, Cunningham ML, Cox TC. Quantitative trait loci affecting the 3D skull shape and size in mouse and prioritization of candidate genes in-silico. Front Physiol. 2015; 6: 92. doi: 10.3389/fphys.2015.00092.

20. Pallares LF, Harr B, Turner LM, Tautz D. Use of a natural hybrid zone for genomewide association mapping of craniofacial traits in the house mouse. Molecular Ecology. 2014; 23: 5756-5770. doi: 10.1111/mec.12968.

21. Pallares LF, Carbonetto P, Gopalakrishnan S, Parker CC, Ackert-Bicknell CL, Palmer AA, et al. Mapping of Craniofacial Traits in Outbred Mice Identifies Major Developmental Genes Involved 
bioRxiv preprint doi: https://doi.org/10.1101/2020.01.24.918011; this version posted June 14,2020 . The copyright holder for this preprint (which was not certified by peer review) is the author/funder, who has granted bioRxiv a license to display the preprint in perpetuity. It is made available under aCC-BY-NC-ND 4.0 International license.

in Shape Determination. PLoS Genetics. 2015; 11: e1005607. doi: 10.1371/journal.pgen.1005607.

22. Arif S, Hilbrant M, Hopfen C, Almudi I, Nunes MDS, Posnien N, et al. Genetic and developmental analysis of differences in eye and face morphology between Drosophila simulans and Drosophila mauritiana. Evolution \& Development. 2013; 15: 257-267. doi: 10.1111/ede.12027.

23. Gaspar P, Arif S, Sumner-Rooney L, Kittelmann M, Bodey AJ, Stern DL, et al. Characterization of the Genetic Architecture Underlying Eye Size Variation Within Drosophila melanogaster and Drosophila simulans. G3 (Bethesda, Md.). 2020; 10: 1005-1018. doi: 10.1534/g3.119.400877.

24. Norry FM, Gomez FH. Quantitative Trait Loci and Antagonistic Associations for Two Developmentally Related Traits in the Drosophila Head. Journal of Insect Science. 2017; 17: 19. doi: 10.1093/jisesa/iew115.

25. Featherstone DE, Broadie K. Wrestling with pleiotropy: genomic and topological analysis of the yeast gene expression network. Bioessays. 2002; 24: 267-274. doi: 10.1002/bies.10054.

26. Ito T, Chiba T, Ozawa R, Yoshida M, Hattori M, Sakaki Y. A comprehensive two-hybrid analysis to explore the yeast protein interactome. Proceedings of the National Academy of Sciences of the United States of America. 2001; 98: 4569-4574. doi: 10.1073/pnas.061034498.

27. Jeong H, Tombor B, Albert R, Oltvai ZN, Barabási AL. The large-scale organization of metabolic networks. Nature Insight Biodiverstiy. 2000; 407: 651-654. doi: 10.1038/35036627.

28. Uetz P, Giot L, Cagney G, Mansfield TA, Judson RS, Knight JR, et al. A comprehensive analysis of protein-protein interactions in Saccharomyces cerevisiae. Nature. 2000; 403: 623-627. doi: 10.1038/35001009.

29. Barabasi, Albert. Emergence of scaling in random networks. Science. 1999; 286: 509-512. doi: 10.1126/science.286.5439.509.

30. Barabási A-L, Oltvai ZN. Network biology: understanding the cell's functional organization. Nat Rev Genet. 2004; 5: 101-113. doi: 10.1038/nrg1272.

31. Fraser HB, Hirsh AE, Steinmetz LM, Scharfe C, Feldman MW. Evolutionary rate in the protein interaction network. Science. 2002; 296: 750-752. doi: 10.1126/science.1068696.

32. Krylov DM, Wolf YI, Rogozin IB, Koonin EV. Gene loss, protein sequence divergence, gene dispensability, expression level, and interactivity are correlated in eukaryotic evolution. Genome Research. 2003; 13: 2229-2235. doi: 10.1101/gr.1589103.

33. Stern DL, Orgogozo V. The loci of evolution: how predictable is genetic evolution. How predictable is genetic evolution. Evolution; International Journal of Organic Evolution. 2008; 62: 2155-2177. doi: 10.1111/j.1558-5646.2008.00450.x.

34. Stern DL, Orgogozo V. Is genetic evolution predictable. Science. 2009; 323: 746-751. doi: $10.1126 /$ science.1158997.

35. Stern DL. PERSPECTIVE. EVOLUTIONARY DEVELOPMENTAL BIOLOGY AND THE PROBLEM OF VARIATION. Evolution; International Journal of Organic Evolution. 2000; 54: 1079. doi: 10.1554/0014-3820(2000)054[1079:PEDBAT]2.0.CO;2.

36. Peter IS, Davidson EH. Evolution of gene regulatory networks controlling body plan development. Cell. 2011; 144: 970-985. doi: 10.1016/j.cell.2011.02.017.

37. Potier D, Davie K, Hulselmans G, Naval Sanchez M, Haagen L, Huynh-Thu VA, et al. Mapping gene regulatory networks in Drosophila eye development by large-scale transcriptome perturbations and motif inference. Cell Reports. 2014; 9: 2290-2303. doi: 10.1016/j.celrep.2014.11.038.

38. Snodgrass RE. Principles of insect morphology. Ithaca, United States: McGraw-Hill Book Co; 1935.

39. Keesey IW, Grabe V, Gruber L, Koerte S, Obiero GF, Bolton G, et al. Inverse resource allocation between vision and olfaction across the genus Drosophila. Nature Communications. 2019; 10: 1162. doi: 10.1038/s41467-019-09087-z. 
bioRxiv preprint doi: https://doi.org/10.1101/2020.01.24.918011; this version posted June 14,2020 . The copyright holder for this preprint (which was not certified by peer review) is the author/funder, who has granted bioRxiv a license to display the preprint in perpetuity. It is made available under aCC-BY-NC-ND 4.0 International license.

40. Norry FM, Vilardi JC, Hasson E. Genetic and phenotypic correlations among size-related traits, and heritability variation between body parts in Drosophila buzzatii. Genetica. 1997; 101: 131139. doi: 10.1023/a:1018360804439.

41. Norry FM, Vilardi JC, Hasson E. Negative genetic correlation between traits of the Drosophila head, and interspecific divergence in head shape. Heredity. 2000; 85: 177-183. doi: 10.1046/j.1365-2540.2000.00735.x.

42. Posnien N, Hopfen C, Hilbrant M, Ramos-Womack M, Murat S, Schönauer A, et al. Evolution of eye morphology and rhodopsin expression in the Drosophila melanogaster species subgroup. PLoS ONE. 2012; 7: e37346. doi: 10.1371/journal.pone.0037346.

43. Hammerle B, Ferrus A. Expression of enhancers is altered in Drosophila melanogaster hybrids. Evol Dev. 2003; 5: 221-230. doi: 10.1046/j.1525-142X.2003.03030.x.

44. Ramaekers A, Claeys A, Kapun M, Mouchel-Vielh E, Potier D, Weinberger S, et al. Altering the Temporal Regulation of One Transcription Factor Drives Evolutionary Trade-Offs between Head Sensory Organs. Developmental Cell. 2019; 50: 780-792.e7. doi: 10.1016/j.devcel.2019.07.027.

45. Haynie JL, Bryant PJ. Development of the eye-antenna imaginal disc and morphogenesis of the adult head in Drosophila melanogaster. The Journal of experimental zoology. 1986; 237: 293308. doi: 10.1002/jez.1402370302.

46. Ostrin EJ, Li Y, Hoffman K, Liu J, Wang K, Zhang L, et al. Genome-wide identification of direct targets of the Drosophila retinal determination protein Eyeless. Genome Research. 2006; 16: 466-476. doi: 10.1101/gr.4673006.

47. Yeung K, Wang F, Li Y, Wang K, Mardon G, Chen R. Integrative genomic analysis reveals novel regulatory mechanisms of eyeless during Drosophila eye development. Nucleic Acids Research. 2018. doi: 10.1093/nar/gky892.

48. Hilbrant M, Almudi I, Leite DJ, Kuncheria L, Posnien N, Nunes MD, et al. Sexual dimorphism and natural variation within and among species in the Drosophila retinal mosaic. BMC evolutionary biology. 2014; 14: 240. doi: 10.1186/s12862-014-0240-x.

49. Torres-Oliva M, Schneider J, Wiegleb G, Kaufholz F, Posnien N. Dynamic genome wide expression profiling of Drosophila head development reveals a novel role of Hunchback in retinal glia cell development and blood-brain barrier integrity. PLoS Genetics. 2018; 14: e1007180. doi: 10.1371/journal.pgen.1007180.

50. Arda HE, Walhout AJM. Gene-centered regulatory networks. Briefings in Functional Genomics. 2010; 9: 4-12. doi: 10.1093/bfgp/elp049.

51. Bar-Joseph Z, Gerber GK, Lee TI, Rinaldi NJ, Yoo JY, Robert F, et al. Computational discovery of gene modules and regulatory networks. Nat Biotechnol. 2003; 21: 1337-1342. doi: $10.1038 / n b t 890$.

52. Segal E, Shapira M, Regev A, Pe'er D, Botstein D, Koller D, et al. Module networks: identifying regulatory modules and their condition-specific regulators from gene expression data. Nat Genet. 2003; 34: 166-176. doi: 10.1038/ng1165.

53. Mishra AK, Bargmann BOR, Tsachaki M, Fritsch C, Sprecher SG. Functional genomics identifies regulators of the phototransduction machinery in the Drosophila larval eye and adult ocelli. Developmental Biology. 2016; 410: 164-177. doi: 10.1016/j.ydbio.2015.12.026.

54. Zheng L, Carthew RW. Lola regulates cell fate by antagonizing Notch induction in the Drosophila eye. Mechanisms of Development. 2008; 125: 18-29. doi: 10.1016/j.mod.2007.10.007.

55. Brennan CA, Ashburner M, Moses K. Ecdysone pathway is required for furrow progression in the developing Drosophila eye. Development. 1998; 125: 2653-2664.

56. Neto M, Naval-Sánchez M, Potier D, Pereira PS, Geerts D, Aerts S, et al. Nuclear receptors connect progenitor transcription factors to cell cycle control. Scientific Reports. 2017; 7: 4845. doi: 10.1038/s41598-017-04936-7. 
57. Sekelsky JJ, Newfeld SJ, Raftery LA, Chartoff EH, Gelbart WM. Genetic characterization and cloning of mothers against dpp, a gene required for decapentaplegic function in Drosophila melanogaster. Genetics. 1995; 139: 1347-1358.

58. Cordero JB, Larson DE, Craig CR, Hays R, Cagan R. Dynamic decapentaplegic signaling regulates patterning and adhesion in the Drosophila pupal retina. Development. 2007; 134: 18611871. doi: $10.1242 / \mathrm{dev} .002972$.

59. Wells BS, Pistillo D, Barnhart E, Desplan C. Parallel Activin and BMP signaling coordinates R7/R8 photoreceptor subtype pairing in the stochastic Drosophila retina. eLife. 2017; 6. doi: 10.7554/eLife.25301.

60. Wiersdorff V, Lecuit T, Cohen SM, Mlodzik M. Mad acts downstream of Dpp receptors, revealing a differential requirement for dpp signaling in initiation and propagation of morphogenesis in the Drosophila eye. Development. 1996; 122: 2153-2162.

61. Maurel-Zaffran C, Treisman JE. pannier acts upstream of wingless to direct dorsal eye disc development in Drosophila. Development. 2000; 127: 1007-1016.

62. Pereira PS, Pinho S, Johnson K, Couso JP, Casares F. A 3' cis-regulatory region controls wingless expression in the Drosophila eye and leg primordia. Dev Dyn. 2006; 235: 225-234. doi: 10.1002/dvdy.20606.

63. Singh A, Choi K-W. Initial state of the Drosophila eye before dorsoventral specification is equivalent to ventral. Development. 2003; 130: 6351-6360. doi: 10.1242/dev.00864.

64. Kitano H. Biological robustness. Nature Reviews Genetics. 2004; 5: 826-837. doi: 10.1007/9783-7643-7567-6_10.

65. Waddington CH. CANALIZATION OF DEVELOPMENT AND THE INHERITANCE OF ACQUIRED CHARACTERS. Nature. 1942; 150: 563-565. doi: 10.1038/150563a0.

66. Cannavò E, Koelling N, Harnett D, Garfield D, Casale FP, Ciglar L, et al. Genetic variants regulating expression levels and isoform diversity during embryogenesis. Nature. 2017; 541: 402-406. doi: 10.1038/nature20802.

67. Fear JM, León-Novelo LG, Morse AM, Gerken AR, van Lehmann K, Tower J, et al. Buffering of Genetic Regulatory Networks in Drosophila melanogaster. Genetics. 2016; 203: 1177-1190. doi: 10.1534/genetics.116.188797.

68. Gavin-Smyth J, Wang YC, Butler I, Ferguson EL. A genetic network conferring canalization to a bistable patterning system in Drosophila. Current Biology. 2013; 23: 2296-2302. doi: 10.1016/j.cub.2013.09.055.

69. Singh A, Chan J, Chern JJ, Choi K-W. Genetic interaction of Lobe with its modifiers in dorsoventral patterning and growth of the Drosophila eye. Genetics. 2005; 171: 169-183. doi: 10.1534/genetics.105.044180.

70. García-García MJ, Ramain P, Simpson P, Modolell J. Different contributions of pannier and wingless to the patterning of the dorsal mesothorax of Drosophila. Development. 1999; 126 : 3523-3532.

71. Oros SM, Tare M, Kango-Singh M, Singh A. Dorsal eye selector pannier (pnr) suppresses the eye fate to define dorsal margin of the Drosophila eye. Developmental Biology. 2010; 346: 258271. doi: 10.1016/j.ydbio.2010.07.030.

72. Murali T, Pacifico S, Yu J, Guest S, Roberts GG, Finley RL. DroID 2011: a comprehensive, integrated resource for protein, transcription factor, RNA and gene interactions for Drosophila. Nucleic Acids Research. 2011; 39: D736-43. doi: 10.1093/nar/gkq1092.

73. Yu J, Pacifico S, Liu G, Finley RL. DroID: the Drosophila Interactions Database, a comprehensive resource for annotated gene and protein interactions. BMC Genomics. 2008; 9: 461. doi: 10.1186/1471-2164-9-461.

74. Cubadda Y, Heitzler P, Ray RP, Bourouis M, Ramain P, Gelbart W, et al. u-shaped encodes a zinc finger protein that regulates the proneural genes achaete and scute during the formation of bristles in Drosophila. Genes \& Development. 1997; 11: 3083-3095. doi: 10.1101/gad.11.22.3083. 
bioRxiv preprint doi: https://doi.org/10.1101/2020.01.24.918011; this version posted June 14,2020 . The copyright holder for this preprint (which was not certified by peer review) is the author/funder, who has granted bioRxiv a license to display the preprint in perpetuity. It is made available under aCC-BY-NC-ND 4.0 International license.

75. Gómez-Skarmeta JL, Campuzano S, Modolell J. Half a century of neural prepatterning: the story of a few bristles and many genes. The story of a few bristles and many genes. Nature reviews. Neuroscience. 2003; 4: 587-598. doi: 10.1038/nrn1142.

76. Heitzler P, Haenlin M, Ramain P, Calleja M, Simpson P. A genetic analysis of pannier, a gene necessary for viability of dorsal tissues and bristle positioning in Drosophila. Genetics. 1996; 143: 1271-1286.

77. Haenlin M, Cubadda Y, Blondeau F, Heitzler P, Lutz Y, Simpson P, et al. Transcriptional activity of Pannier is regulated negatively by heterodimerization of the GATA DNA-binding domain with a cofactor encoded by the u-shaped gene of Drosophila. Genes \& Development. 1997; 11: 30963108. doi: 10.1101/gad.11.22.3096.

78. Fossett N, Tevosian SG, Gajewski K, Zhang Q, Orkin SH, Schulz RA. The Friend of GATA proteins U-shaped, FOG-1, and FOG-2 function as negative regulators of blood, heart, and eye development in Drosophila. Proceedings of the National Academy of Sciences. 2001; 98: 73427347. doi: 10.1073/pnas.131215798.

79. Sorrentino RP, Tokusumi T, Schulz RA. The Friend of GATA protein U-shaped functions as a hematopoietic tumor suppressor in Drosophila. Developmental Biology. 2007; 311: 311-323. doi: 10.1016/j.ydbio.2007.08.011.

80. Casares F, Almudi I. Fast and Furious 800. The Retinal Determination Gene Network in Drosophila. In: Castelli-Gair Hombría J, Bovolenta P, editors. Organogenetic Gene Networks. Genetic Control of Organ Formation. Cham, s.l.: Springer International Publishing; 2016. pp. 95124.

81. Baker LR, Weasner BM, Nagel A, Neuman SD, Bashirullah A, Kumar JP. Eyeless/Pax6 initiates eye formation non-autonomously from the peripodial epithelium. Development (Cambridge, England). 2018; 145. doi: 10.1242/dev.163329.

82. Magri MS, Domínguez-Cejudo MA, Casares F. Wnt controls the medial-lateral subdivision of the Drosophila head. Biol Lett. 2018; 14. doi: 10.1098/rsbl.2018.0258.

83. Wang LH, Huang YT, Tsai YC, Sun YH. The role of eyg Pax gene in the development of the head vertex in Drosophila. Developmental Biology. 2010; 337: 246-258. doi: 10.1016/j.ydbio.2009.10.038.

84. Fromental-Ramain C, Vanolst L, Delaporte C, Ramain P. pannier encodes two structurally related isoforms that are differentially expressed during Drosophila development and display distinct functions during thorax patterning. Mechanisms of Development. 2008; 125: 43-57. doi: 10.1016/j.mod.2007.10.008.

85. Fromental-Ramain C, Taquet N, Ramain P. Transcriptional interactions between the pannier isoforms and the cofactor U-shaped during neural development in Drosophila. Mechanisms of Development. 2010; 127: 442-457. doi: 10.1016/j.mod.2010.08.002.

86. Lachaise D, David JR, Lemeunier F, Tsacas L. The reproductive relationships of Drosophila sechellia with D. mauritiana, D. simulans, and D. melanogaster from the Afrotropical region. Evolution. 1986; 40: 262-271.

87. Stern DL. Identification of loci that cause phenotypic variation in diverse species with the reciprocal hemizygosity test. Trends in Genetics. 2014; 30: 547-554. doi: 10.1016/j.tig.2014.09.006.

88. Wagner GP, Zhang J. The pleiotropic structure of the genotype-phenotype map: the evolvability of complex organisms. Nature reviews. Genetics. 2011; 12: 204-213. doi: 10.1038/nrg2949.

89. Batada NN, Hurst LD. Evolution of chromosome organization driven by selection for reduced gene expression noise. Nat Genet. 2007; 39: 945-949. doi: 10.1038/ng2071.

90. Landry CR, Lemos B, Rifkin Sa, Dickinson WJ, Hartl DL. Genetic properties influencing the evolvability of gene expression. Science. 2007; 317: 118-121. doi: 10.1126/science.1140247.

91. Lehner B. Selection to minimise noise in living systems and its implications for the evolution of gene expression. Mol Syst Biol. 2008; 4: 170. doi: 10.1038/msb.2008.11. 
92. Huang F, Dambly-Chaudière C, Ghysen A. The emergence of sense organs in the wing disc of Drosophila. Development. 1991; 111: 1087-1095.

93. Kernan MJ. Mechanotransduction and auditory transduction in Drosophila. Pflugers Arch. 2007; 454: 703-720. doi: 10.1007/s00424-007-0263-x.

94. Matamoro-Vidal A, Tully T, Courtier-Orgogozo V. Robustness of bristle number to temperature and genetic background varies with bristle type and is regulated by miR-9a. bioRxiv. 2018 . doi: $10.1101 / 295485$.

95. Cagan RL, Ready DF. The emergence of order in the Drosophila pupal retina. Developmental Biology. 1989; 136: 346-362. doi: 10.1016/0012-1606(89)90261-3.

96. Bonini NM, Bui QT, Gray-Board GL, Warrick JM. The Drosophila eyes absent gene directs ectopic eye formation in a pathway conserved between flies and vertebrates. Development. 1997; 124: 4819-4826.

97. Halder G, Callaerts P, Gehring WJ. Induction of ectopic eyes by targeted expression of the eyeless gene in Drosophila. Science (New York, N.Y.). 1995; 267: 1788-1792. doi: 10.1126/science.7892602.

98. Pan D, Rubin GM. Targeted expression of teashirt induces ectopic eyes in Drosophila. Proceedings of the National Academy of Sciences of the United States of America. 1998; 95 : 15508-15512. doi: 10.1073/pnas.95.26.15508.

99. Seimiya M, Gehring WJ. The Drosophila homeobox gene optix is capable of inducing ectopic eyes by an eyeless-independent mechanism. Development. 2000; 127: 1879-1886.

100. Shen W, Mardon G. Ectopic eye development in Drosophila induced by directed dachshund expression. Development. 1997; 124: 45-52.

101. Alvarez-Ponce D, Aguadé M, Rozas J. Network-level molecular evolutionary analysis of the insulin/TOR signal transduction pathway across 12 Drosophila genomes. Genome Research. 2009; 19: 234-242. doi: 10.1101/gr.084038.108.

102. Davila-Velderrain J, Servin-Marquez A, Alvarez-Buylla ER. Molecular evolution constraints in the floral organ specification gene regulatory network module across 18 angiosperm genomes. Molecular Biology and Evolution. 2014; 31: 560-573. doi: 10.1093/molbev/mst223.

103. Wagner GP, Lynch VJ. The gene regulatory logic of transcription factor evolution. Trends in Ecology \& Evolution. 2008; 23: 377-385. doi: 10.1016/j.tree.2008.03.006.

104. Borneman AR, Leigh-Bell JA, Yu H, Bertone P, Gerstein M, Snyder M. Target hub proteins serve as master regulators of development in yeast. Genes \& Development. 2006; 20: 435-448. doi: 10.1101/gad.1389306.

105. Vermeirssen V, Barrasa MI, Hidalgo CA, Babon JAB, Sequerra R, Doucette-Stamm L, et al. Transcription factor modularity in a gene-centered C. elegans core neuronal protein-DNA interaction network. Genome Research. 2007; 17: 1061-1071. doi: 10.1101/gr.6148107.

106. Promislow D. A regulatory network analysis of phenotypic plasticity in yeast. The American naturalist. 2005; 165: 515-523. doi: 10.1086/429161.

107. Cai JJ, Borenstein E, Petrov DA. Broker genes in human disease. Genome Biology and Evolution. 2010; 2: 815-825. doi: 10.1093/gbe/evq064.

108. Macneil LT, Walhout AJM. Gene regulatory networks and the role of robustness and stochasticity in the control of gene expression. Genome Research. 2011; 21: 645-657. doi: 10.1101/gr.097378.109.

109. Bessa J, Gebelein B, Pichaud F, Casares F, Mann RS. Combinatorial control of Drosophila eye development by eyeless, homothorax, and teashirt. Genes \& Development. 2002; 16: 2415-2427. doi: 10.1101/gad.1009002.

110. Lopes CS, Casares F. Hth maintains the pool of eye progenitors and its downregulation by Dpp and Hh couples retinal fate acquisition with cell cycle exit. Developmental Biology. 2010; 339: 78-88. doi: 10.1016/j.ydbio.2009.12.020.

111. Treisman JE. Retinal differentiation in Drosophila. Wiley Interdisciplinary Reviews: Developmental Biology. 2013; 2: 545-557. doi: 10.1002/wdev.100. 
bioRxiv preprint doi: https://doi.org/10.1101/2020.01.24.918011; this version posted June 14,2020 . The copyright holder for this preprint (which was not certified by peer review) is the author/funder, who has granted bioRxiv a license to display the preprint in perpetuity. It is made available under aCC-BY-NC-ND 4.0 International license.

112. Palliyil S, Zhu J, Baker LR, Neuman SD, Bashirullah A, Kumar JP. Allocation of distinct organ fates from a precursor field requires a shift in expression and function of gene regulatory networks. PLoS Genetics. 2018; 14: e1007185. doi: 10.1371/journal.pgen.1007185.

113. Buchberger E, Reis M, Lu T-H, Posnien N. Cloudy with a Chance of Insights: Context Dependent Gene Regulation and Implications for Evolutionary Studies. Genes (Basel). 2019; 10. doi: 10.3390/genes10070492.

114. Costanzo M, Baryshnikova A, Bellay J, Kim Y, Spear ED, Sevier CS, et al. The genetic landscape of a cell. Science (New York, N.Y.). 2010; 327: 425-431. doi: 10.1126/science.1180823.

115. Frankel N, Davis GK, Vargas D, Wang S, Payre F, Stern DL. Phenotypic robustness conferred by apparently redundant transcriptional enhancers. Nature. 2010; 466: 490-493. doi: 10.1038/nature09158.

116. Giaever G, Chu AM, Ni L, Connelly C, Riles L, Véronneau S, et al. Functional profiling of the Saccharomyces cerevisiae genome. Nature. 2002; 418: 387-391. doi: 10.1038/nature00935.

117. Hollenhorst PC, Shah AA, Hopkins C, Graves BJ. Genome-wide analyses reveal properties of redundant and specific promoter occupancy within the ETS gene family. Genes \& Development. 2007; 21: 1882-1894. doi: 10.1101/gad.1561707.

118. Kamath RS, Fraser AG, Dong Y, Poulin G, Durbin R, Gotta M, et al. Systematic functional analysis of the Caenorhabditis elegans genome using RNAi. Nature. 2003; 421: 231-237. doi: 10.1038/nature01278.

119. Tsai A, Alves MR, Crocker J. Multi-enhancer transcriptional hubs confer phenotypic robustness. eLife. 2019; 8. doi: 10.7554/eLife.45325.

120. Chen C, Zhang D, Hazbun TR, Zhang M. Inferring Gene Regulatory Networks from a Population of Yeast Segregants. Sci Rep. 2019; 9: 1197. doi: 10.1038/s41598-018-37667-4.

121. Thompson D, Regev A, Roy S. Comparative analysis of gene regulatory networks: from network reconstruction to evolution. Annual Review of Cell and Developmental Biology. 2015; 31: 399428. doi: 10.1146/annurev-cellbio-100913-012908.

122. Tsuda ME, Kawata M. Evolution of gene regulatory networks by fluctuating selection and intrinsic constraints. PLOS Computational Biology. 2010; 6. doi: 10.1371/journal.pcbi.1000873.

123. Langmead B, Salzberg SL. Fast gapped-read alignment with Bowtie 2. Nat Methods. 2012; 9: 357-359. doi: 10.1038/nmeth.1923.

124. Li H, Handsaker B, Wysoker A, Fennell T, Ruan J, Homer N, et al. The Sequence Alignment/Map format and SAMtools. Bioinformatics. 2009; 25: 2078-2079. doi: 10.1093/bioinformatics/btp352.

125. Li H. A statistical framework for SNP calling, mutation discovery, association mapping and population genetical parameter estimation from sequencing data. Bioinformatics. 2011; 27: 2987-2993. doi: 10.1093/bioinformatics/btr509.

126. Love MI, Huber W, Anders S. Moderated estimation of fold change and dispersion for RNA-seq data with DESeq2. Genome Biology. 2014; 15: 550. doi: 10.1186/s13059-014-0550-8.

127. Godichon-Baggioni A, Maugis-Rabusseau C, Rau A. Clustering transformed compositional data using $\mathrm{K}$-means, with applications in gene expression and bicycle sharing system data. Journal of Applied Statistics. 2019; 46: 47-65. doi: 10.1080/02664763.2018.1454894.

128. Rau A, Maugis-Rabusseau C. Transformation and model choice for RNA-seq co-expression analysis. Briefings in bioinformatics. 2018; 19: 425-436. doi: 10.1093/bib/bbw128.

129. Herrmann C, van de Sande B, Potier D, Aerts S. i-cisTarget. An integrative genomics method for the prediction of regulatory features and cis-regulatory modules. Nucleic Acids Research. 2012; 40: e114-e114. doi: 10.1093/nar/gks543.

130. Imrichová H, Hulselmans G, Atak ZK, Potier D, Aerts S. i-cisTarget 2015 update. Generalized cis-regulatory enrichment analysis in human, mouse and fly. Nucleic Acids Research. 2015; 43: W57-64. doi: 10.1093/nar/gkv395. 
bioRxiv preprint doi: https://doi.org/10.1101/2020.01.24.918011; this version posted June 14, 2020. The copyright holder for this preprint (which was not certified by peer review) is the author/funder, who has granted bioRxiv a license to display the preprint in perpetuity. It is made available under aCC-BY-NC-ND 4.0 International license.

1 131. Buenrostro JD, Giresi PG, Zaba LC, Chang HY, Greenleaf WJ. Transposition of native 2 chromatin for fast and sensitive epigenomic profiling of open chromatin, DNA-binding proteins and nucleosome position. Nature Methods. 2013; 10: 1213-1218. doi: 10.1038/nmeth.2688.

132. Junion G, Spivakov M, Girardot C, Braun M, Gustafson EH, Birney E, et al. A transcription factor collective defines cardiac cell fate and reflects lineage history. Cell. 2012; 148: 473-486. doi: 10.1016/j.cell.2012.01.030. 